\title{
Optimal Walking Gait Generator for Biped Robot Using Modified Jaya Optimization Technique
}

\author{
Ho Pham Huy Anh ${ }^{1, *}$, Tran Thien Huan ${ }^{2}$ \\ ${ }^{1}$ Faculty of Electrical and Electronics Engineering (FEEE), HCM City University of Technology, VNU-HCM, Ho Chi Minh City, Vietnam \\ ${ }^{2}$ Faculty of Applied Sciences (FAS), HCM City University of Technology and Education, Ho Chi Minh City, Vietnam
}

\section{ARTICLE INFO \\ Article History \\ Received 23 Sep 2019 \\ Accepted 03 Mar 2020 \\ Keywords \\ Uncertain nonlinear biped robot system \\ Genetic Algorithm (GA) \\ Particle Swarm Optimization (PSO) \\ Jaya optimization algorithm \\ Central Force Optimization (CFO) \\ algorithm \\ Modified Differential Evolution \\ (MDE) algorithm \\ Gait optimization for biped robot \\ Zero moment point (ZMP) concept}

\begin{abstract}
This paper treats the optimization of the biped walking trajectory that can be used as a reference trajectory for control. The biped robot is modeled as a kinetic chain of 11 links connected by 10 joints. The inverse kinematics of the biped is derived for the specified positions of the hips and feet. The objective is to optimize the biped robot able to stably and naturally walking with preset foot-lift magnitude (or preset hip-shift, or preset step-length). The stability of the biped robot is quantified by the distance between the ZMP and the foot center in the step cycle, which represents the first objective function. Additionally, for the biped robot to follow the preset foot-lift value, the difference between the magnitude of foot-lift value and the foot-lift preset value represents the second objective function. Specifically, we minimize the value of the two objective functions by considering the gait parameters of biped robot as variables. The new Jaya optimization algorithm is innovatively applied to optimize the biped gait parameters as to ensure the biped robot walking robustly and steadily. The efficiency of the proposed Jaya-based identification method is compared with the Genetic Algorithm (GA), the Particle Swarm Optimization (PSO), the Central Force Optimization (CFO) and improved Differential Evolution (DE) [Modified Differential Evolution (MDE)] algorithms. The simulation results tested on the real small-sized biped robot system HUBOT-4 demonstrate that the novel proposed algorithm offers an efficient and stable gait for biped robot with precise foot-lift value.
\end{abstract}

(C) 2020 The Authors. Published by Atlantis Press SARL. This is an open access article distributed under the CC BY-NC 4.0 license (http://creativecommons.org/licenses/by-nc/4.0/).

\section{INTRODUCTION}

The advanced biped robot walking mechanism is increasingly researched and developed in various aspects. Novel standards have been verified and applied to humanoid robots as to ensure more stable and natural gait gesture. Static stable walking is the first applied principle, in which the vertical projection of center of mass (CoM) on the ground is always to be kept within the supporting foot surface. In other words, humanoid robots can stop and move at any times during walking without falling apart. Based on its very simple manner, this principle is only successfully applied to slowspeed biped robots in which dynamic effects can be ignored [1,2]. Researchers then began to focus on developing dynamic walking [3]. This method allows the humanoid robot to speed up the pace. However, during locomotion process, the biped may fall due to environmental interference and cannot stop abruptly. Therefore, Zero Moment Point (ZMP)-based biped walking was proposed to help control and to handle biped inertia $[4,5]$.

Numerous interesting researches have focused on improving the performance of humanoid robot walking gesture. Huang in [6]

\footnotetext{
${ }^{*}$ Corresponding author. Email: hophamhuyanh@hotmail.com
}

proposed a stable gait generator based on a newly proposed gait pattern in using the interpolation function. The method developed by D. Huan, through the Genetic Algorithm (GA), optimized the gait generator parameters as to help robot move steadily with the least amount of energy [7]. Dip et al. [8] exhibited steady gait for biped at constant velocity using the sine wave generator. Maximo et al. [9] introduced a new stable and quick model-free gait with specific arms movement for biped robots. Khusainov et al. [10] successfully combined kinematics and dynamics approaches in gait optimization for humanoid robot locomotion. The fact is that, nowadays, intelligent algorithms are being increasingly applied in this domain to optimize the gait generator parameters for humanoid robots such as GA [8], Particle Swarm Optimization (PSO) algorithm [11], Modified Differential Evolution (MDE) algorithm [12], Central Force Optimization (CFO) algorithm [13]. Shaffi in [14] introduced the humanoid robot achieved a stable gait by using the Fourier seriesbased gait generator. Furthermore novel approaches continually applied different intelligent algorithms to develop human robot stable and nature walking movement, such as bee swarm algorithm [15], T-S fuzzy controller [16], evolution techniques [17], recurrent neural networks optimized by Ant-Colony Optimization (ACO) [18] and so on. These intelligent algorithms have shown 
their effectiveness in gait generator for humanoid robot. However, there are still numerous areas for further improvement, especially those related to the computational cost of meta-heuristic algorithms.

Recently among newly proposed meta-heuristic optimization approaches, the powerful potential of the Jaya optimization algorithm has not yet being applied to optimize the biped robot walking gait generator. An enhanced optimization method, namely Jaya, initiatively introduced by Rao [19], proves compact and efficient to use with great benefit related to no need any particular control coefficients. Thus, it has been quickly used and improved for optimally solving different problems in various sectors such as mechatronics (Kumar et al. [20]; Rao et al. [21]), neural model identification (Suraj and Ghosh [22]), electronics (Bhoye et al. [23]), optimal civil problems (Venkata Rao and Saroj [24]; Du et al. [25]; Cong et al. [26]), control system implementation (Rao and More [27]; Rao and Saroj [28]) among others.

Enhancing with promising applications of the Jaya optimization algorithm, this paper intends to improve the modified Jaya approach for proposing a new approach of optimal nature-walking gait applied to biped robot.

In this paper, a new nature-walking pattern generation ( $n$-WPG) is proposed which consists of gait generator for the two-foot trajectory, hip trajectory and the inverse kinematics in the full walking periodic sequence which can be seen as combination of three sub-sequences: Starting step, Periodic steps and Ending step. A combined objective function is newly introduced by linking two separate objective functions for humanoid robots that allows stable stepping with preset foot-lifting magnitude. The first one is based on ZMP criterion to stable stepping and the second is based on the difference between the magnitude of the foot-lift parameter and the foot-lift preset value. The Jaya algorithm is used to optimally identify the gait parameters for the biped as to reach steady nature-walking gait with accurate preset foot-lifting magnitude.
The simulation and experiment results of proposed algorithm applied on the small-sized biped robot demonstrate the performance of the novel algorithm allowing the biped robot to move steadily and stably with an effectively reduced training time. The optimal nature-walking gait results attained by the proposed algorithm are comparatively tested with those obtained by well-known optimization approaches such as Modified Differential Evolution (MDE), CFO, GA and PSO algorithms.

The rest of this paper is arranged as follows: Section 2 fully presents the model design and implementation of the biped robot. In Section 3, inverse kinematics applied in biped optimized WPG parameters is presented. Section 4 presents the optimization results of the biped robot gait trajectory using Jaya technique in comparison with other four EAs algorithms. Section 5 presents the three Case Studies results. Finally, concluding remarks are given in Section 6.

\section{BIPED MODEL IMPLEMENTATION}

The analysis described in this research is based on a biped robot model shown in Figure 1. The origin of the fixed base reference coordinate $\mathrm{B}$ is located under Leg 1 on the ground plane. The $\mathrm{x}$-axis points forward, the $\mathrm{z}$-axis points upward and the $y$-axis is the cross product of $\mathrm{z}$ and $\mathrm{x}$ axes. The biped robot can be modeled as a kinetic chain of $(2 n+1)$ links connected by $2 n$ revolute joints, where $n=5$ is the number of degrees of freedom of each leg. Figure 1 shows the link and joint coordinate assignments and the values of physical parameters of the biped robot model are shown in Table 1.

The joint angle $i$ is the rotation of frame $i$ relative to frame $(i-1)$ about axis $z_{i}$. Each link has its mass $m_{i}$ concentrated at its CoM $P \mathrm{i}=\left(P_{\mathrm{ix}}, P_{\mathrm{yi}}, P_{\mathrm{zi}}\right)$. The position of each link is computed from the 10 angles of rotation at one time in one step with the original coordination at the center of the supporting foot by direct kinematics, as Equations (1-3).

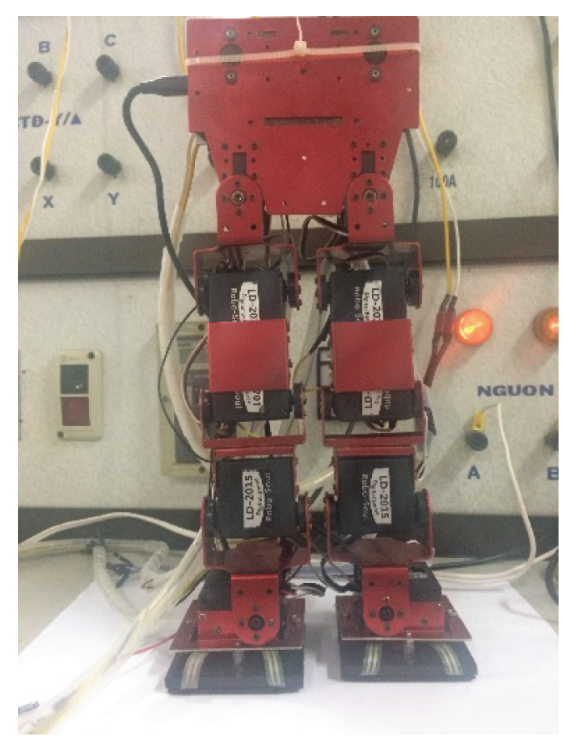

(a)

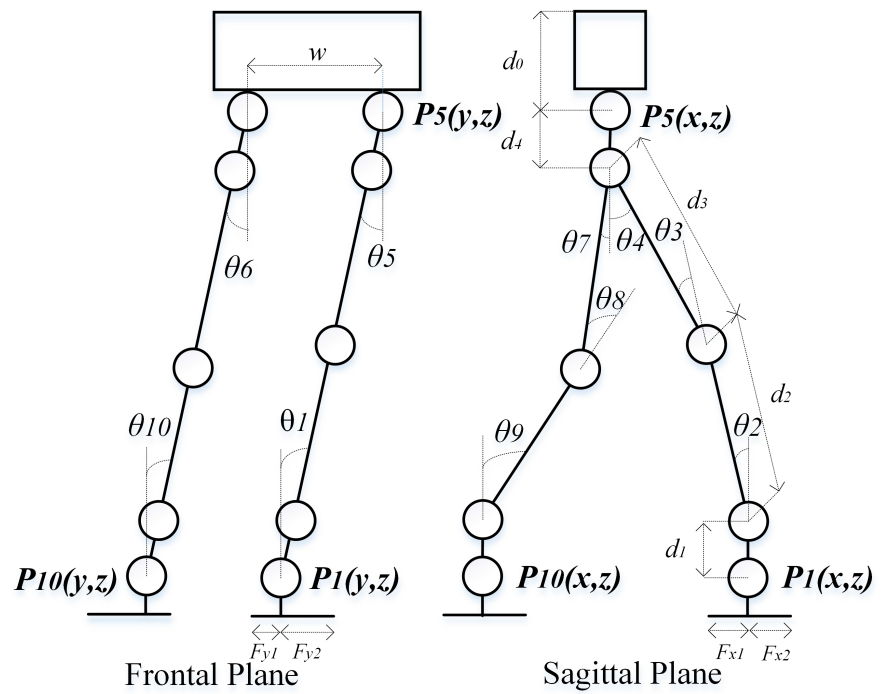

(b)

Figure 1 Biped robot model and coordinate configuration. 
Table 1 Physical parameters of biped robot (HUBOT-4).

\begin{tabular}{ll}
\hline Parameters & Value \\
\hline$d_{0}$ & $6.0 \mathrm{~cm}$ \\
$d_{1}$ & $4.5 \mathrm{~cm}$ \\
$d_{2}$ & $6.0 \mathrm{~cm}$ \\
$d_{3}$ & $6.0 \mathrm{~cm}$ \\
$d_{4}$ & $4.5 \mathrm{~cm}$ \\
$w$ & $6.6 \mathrm{~cm}$ \\
$F_{y 1}$ & $2.0 \mathrm{~cm}$ \\
$F_{y 2}$ & $4.0 \mathrm{~cm}$ \\
$F_{x 1}=F_{x 2}$ & $2.4 \mathrm{~cm}$ \\
$m_{i}$ & $70 \mathrm{~g}$ \\
$i=1 \ldots 11$ & \\
\hline
\end{tabular}

$$
\left\{\begin{array}{l}
P_{1 x t}(t)=0, P_{1 y t}(t)=0, P_{1 z t}(t)=0, \\
P_{2 x t}(t)=P_{1 x t}, P_{2 z t}(t)=d_{1} \cos \left[\theta_{1}(t)\right], \\
P_{2 y t}(t)=P_{2 z t}(t) \sin \left[\theta_{1}(t)\right], \\
P_{3 x t}(t)=d_{2} \sin \left[\theta_{2}(t)\right] \\
P_{3 y t}(t)=P_{3 z t}(t) \sin \left[\theta_{1}(t)\right] \\
P_{3 z t}(t)=P_{2 z t}(t)+d_{2} \cos \left[\theta_{2}(t)\right] \cos \left[\theta_{1}(t)\right], \\
P_{4 x t}(t)=P_{3 x t}(t)+d_{3} \sin \left[\theta_{2}(t)-\theta_{3}(t)\right], \\
P_{4 y t}(t)=P_{4 z t}(t) \sin \left[\theta_{1}(t)\right], \\
P_{4 z t}(t)=P_{3 z t}(t)+d_{3} \cos \left[\theta_{2}(t)-\theta_{3}(t)\right] \cos \left[\theta_{1}(t)\right], \\
P_{5 x t}(t)=P_{4 x t}(t)+d_{4} \sin \left[\theta_{2}(t)-\theta_{3}(t)+\theta_{5}(t)\right], \\
P_{5 y t}(t)=P_{5 z t}(t) \sin \left[\theta_{1}(t)\right], \\
P_{5 z t}(t)=P_{4 z t}(t)+d_{4} \cos \left[\theta_{2}(t)-\theta_{3}(t)+\theta_{5}(t)\right] \cos \left[\theta_{1}(t)\right]
\end{array}\right.
$$

$$
\left\{\begin{array}{l}
P_{5 z t}(t)=P_{4 z t}(t)+d_{4} \cos \left[\theta_{2}(t)-\theta_{3}(t)+\theta_{5}(t)\right] \cos \left[\theta_{1}(t)\right], \\
P_{6 x t}(t)=P_{5 x t}(t), P_{6 y t}(t)=P_{5 y t}(t)-w, P_{6 z t}(t)=P_{5 z t}(t), \\
P_{7 x t}(t)=P_{6 x t}(t), P_{7 z t}(t)=P_{6 z t}(t)-d_{4} \cos \left[\theta_{6}(t)\right], \\
P_{7 y t}(t)=P_{6 y t}(t)-\left[P_{6 z t}(t)-P_{7 z t}(t)\right] \sin \left[\theta_{6}(t)\right], \\
P_{8 x t}(t)=P_{7 x t}(t)+d_{3} \sin \left[\theta_{7}(t)\right], \\
P_{8 z t}(t)=P_{7 z t}(t)-d_{3} \cos \left[\theta_{7}(t)\right] \cos \left[\theta_{6}(t)\right], \\
P_{8 y t}(t)=P_{7 y t}(t)-\left[P_{7 z t}(t)-P_{8 z t}(t)\right] \sin \left[\theta_{6}(t)\right], \\
P_{9 x t}(t)=P_{8 x t}(t)+d_{2} \sin \left[\theta_{7}(t)-\theta_{8}(t)\right], \\
P_{9 z t}(t)=P_{8 z t}(t)-d_{2} \cos \left[\theta_{7}(t)-\theta_{8}(t)\right] \cos \left[\theta_{6}(t)\right], \\
P_{9 y t}(t)=P_{7 y t}(t)-\left[P_{7 z t}(t)-P_{9 z t}(t)\right] \sin \left[\theta_{6}(t)\right], \\
P_{10 x t}(t)=P_{11 x t}(t)+d_{1} \sin \left[\theta_{7}(t)-\theta_{8}(t)+\theta_{1}(t)\right], \\
P_{10 z t}(t)=P_{9 z t}(t)-d_{1} \cos \left[\theta_{7}(t)-\theta_{8}(t)+\theta_{9}(t)\right] \cos \left[\theta_{6}(t)\right], \\
P_{10 y t}(t)=P_{7 y t}(t)-\left[P_{7 z t}(t)-P_{10 z t}(t)\right] \sin \left[\theta_{6}(t)\right]
\end{array}\right.
$$

$$
\left\{\begin{array}{l}
P_{0 x t}(t)=\frac{P_{5 x t}(t)+P_{6 x t}(t)}{2} \\
P_{1 y t}(t)=\frac{P_{5 y t}(t)+P_{6 y t}(t)}{2} \\
P_{0 z t}(t)=P_{5 z t}(t)+\frac{d_{0}}{2}
\end{array}\right.
$$

in which, $d_{0}, d_{1}, d_{2}, d_{3}$ and $d_{4}$ are fully illustrated in Figure 1.

The balancing of the biped system can be simplified at an assigned ground point, called the $\mathrm{ZMP}\left(\mathrm{x}_{Z M P}, \mathrm{y}_{Z M P}, \mathrm{z}_{Z M P}=0\right)$, where the resultant moment at the ground plane is zero. The location of the ZMP [5] is

$$
\left\{\begin{array}{c}
x_{Z M P}=\frac{\sum_{i=1}^{n} m_{i}\left(\ddot{z}_{i}+g\right) x_{i}-\sum_{i=1}^{n} m_{i} \ddot{x}_{i} z_{i}}{\sum_{i=1}^{n} m_{i}\left(\ddot{z}_{i}+g\right)} \\
y_{Z M P}=\frac{\sum_{i=1}^{n} m_{i}\left(\ddot{z}_{i}+g\right) y_{i}-\sum_{i=1}^{n} m_{i} \ddot{y}_{i} z_{i}}{\sum_{i=1}^{n} m_{i}\left(\ddot{z}_{i}+g\right)}
\end{array}\right.
$$

Constraints on the motion of the biped robot are that the location of the ZMP must be inside the convex hull of the support foot or feet. We assume the frictional coefficient is large enough so that the frictional constraint is negligible.

\section{INVERSE KINEMATICS}

To describe the specific configuration of the biped robot, we assign the motions of link 1 (foot 1), link 6 (body) and link 11 (foot 2). By fixing both the position and the orientation of these three links, the configuration of the biped robot is established. For simplicity, we assume that no rotational motion occurs for these three links during walking. In order to compute the joint motion, the inverse kinematic model is needed for given $P_{1}=\left(P_{1 \mathrm{x}}, P_{1 \mathrm{y}}, P_{1 \mathrm{z}}\right), P_{5}=\left(P_{5 \mathrm{x}}\right.$, $\left.P_{5 \mathrm{y}}, P_{5 \mathrm{z}}\right)$ and $P_{10}=\left(P_{10 \mathrm{x}}, P_{10 \mathrm{y}}, P_{10 \mathrm{z}}\right)$ where $P_{\mathrm{i}}$ is the reference point of link $i$ as shown in Figure 1. Because there is no rotation, the joint angles can be expressed by,

$$
\left\{\begin{array}{l}
\theta_{1}(t)=\arctan \left(\frac{y_{l}(t)}{z_{l}(t)}\right), \quad \theta_{5}(t)=-\theta_{1}(t) \\
\theta_{10}(t)=\arctan \left(\frac{y_{r}(t)}{z_{r}(t)}\right), \quad \theta_{6}(t)=-\theta_{10}(t) \\
\theta_{3}(t)=\pi-\theta_{A}(t), \quad \theta_{8}(t)=\pi-\theta_{C}(t) \\
\theta_{4}(t)=\frac{\pi}{2}-\theta_{A}(t)+\theta_{B}(t)-\arcsin \left(\frac{x_{l}(t)}{l_{l}(t)}\right) \\
\theta_{7}(t)=\frac{\pi}{2}-\theta_{C}(t)+\theta_{D}(t)-\arcsin \left(\frac{x_{r}(t)}{l_{r}(t)}\right) \\
\theta_{2}(t)=\theta_{3}(t)-\theta_{4}(t), \quad \theta_{9}(t)=\theta_{8}(t)-\theta_{7}(t)
\end{array}\right.
$$

where

$$
\left\{\begin{array}{l}
x_{l}=P_{5 x}-P_{1 x}, \quad y_{l}=P_{5 y}, \quad z_{l}=P_{5 z}-P_{1 z}, \\
l_{l}=\sqrt{\left(P_{4 x}-P_{2 x}\right)^{2}+\left(P_{4 y}-P_{2 y}\right)^{2}+\left(P_{4 z}-P_{2 z}\right)^{2}}, \\
x_{r}=P_{6 x}-P_{10 x}, \quad y_{r}=P_{5 y}, \quad z_{r}=P_{6 z}-P_{10 z}, \\
l_{r}=\sqrt{\left(P_{7 x}-P_{9 x}\right)^{2}+\left(P_{7 y}-P_{9 y}\right)^{2}+\left(P_{7 z}-P_{9 z}\right)^{2}} \\
\theta_{A}=\arccos \left(\frac{d_{2}^{2}+d_{3}^{2}-l_{l}^{2}}{2 d_{2} d_{3}}\right), \quad \theta_{B}=\arccos \left(\frac{d_{3} \sin \left(\theta_{A}\right)}{l_{l}}\right), \\
\theta_{C}=\arccos \left(\frac{d_{2}^{2}+d_{3}^{2}-l_{r}^{2}}{2 d_{2} d_{3}}\right), \quad \theta_{D}=\arccos \left(\frac{d_{3} \sin \left(\theta_{C}\right)}{l_{l}}\right)
\end{array}\right.
$$


The coordination $P_{6}(x, y, z)$ is computed using $P_{5}(x, y, z)$, and the coordination of $\left[P_{2}(x, y, z), P_{4}(x, y, z), P_{7}(x, y, z), P_{9}(x, y, z)\right]$ is computed using $\left[P_{1}(x, y, z), P_{5}(x, y, z), P_{6}(x, y, z), P_{10}(x, y, z)\right]$ and the rotary angle $\left[\theta_{1}, \theta_{5}, \theta_{6}, \theta_{10}\right]$. Equation (6) below are used to determine $P_{2}, P_{4}, P_{6}, P_{7}, P_{9}$.

$$
\left\{\begin{array}{l}
P_{2 x}=P_{1 x}, P_{2 z}=d_{1} \cos \left(\theta_{1}\right), P_{2 y}=P_{2 z} \sin \left(\theta_{1}\right) \\
P_{4 x}=P_{5 x}, P_{4 z}=P_{5 z}-d_{4} \cos \left(\theta_{1}\right) \\
P_{4 y}=P_{5 y}-\left(P_{5 z}-P_{4 z}\right) \sin \left(\theta_{1}\right) \\
P_{6 x}=P_{5 x}, P_{6 y}=P_{5 y}-w, P_{6 z}=P_{5 z} \\
P_{7 x}=P_{6 x}, P_{7 z}=P_{6 z}-d_{4} \cos \left(\theta_{10}\right) \\
P_{7 y}=P_{6 y}-\left(P_{6 z}-P_{7 z}\right) \sin \left(\theta_{10}\right) \\
P_{9 x}=P_{10 x}, P_{9 z}=P_{10 z}+d_{4} \cos \left(\theta_{10}\right) \\
P_{9 y}=P_{10 y}+\left(P_{9 z}-P_{10 z}\right) \sin \left(\theta_{10}\right)
\end{array}\right.
$$

In that, $d_{0}$ represents the length of the upper body, $d_{1}$ is the distance between two points $P_{1}$ and $P_{2}, d_{2}$ is the distance between two points $P_{2}$ and $P_{3}, d_{3}$ is the distance between two points $P_{3}$ and $P_{4}$, $d_{4}$ represents the distance between two points $P_{4}$ and $P_{5}, w$ represents the distance between 2 legs. Therefore, the biped motion can be expressed solely by positions $P_{1}, P_{5}$ and $P_{10}$. A flowchart for computing the ZMP from the trajectories of $P_{1}, P_{5}$ and $P_{10}$ is shown in Figure 2.

\section{OPTIMIZATION OF THE BIPED ROBOT TRAJECTORY}

The trajectory optimization is based on an objective functional, involving the biped robot able to stably and naturally walking with

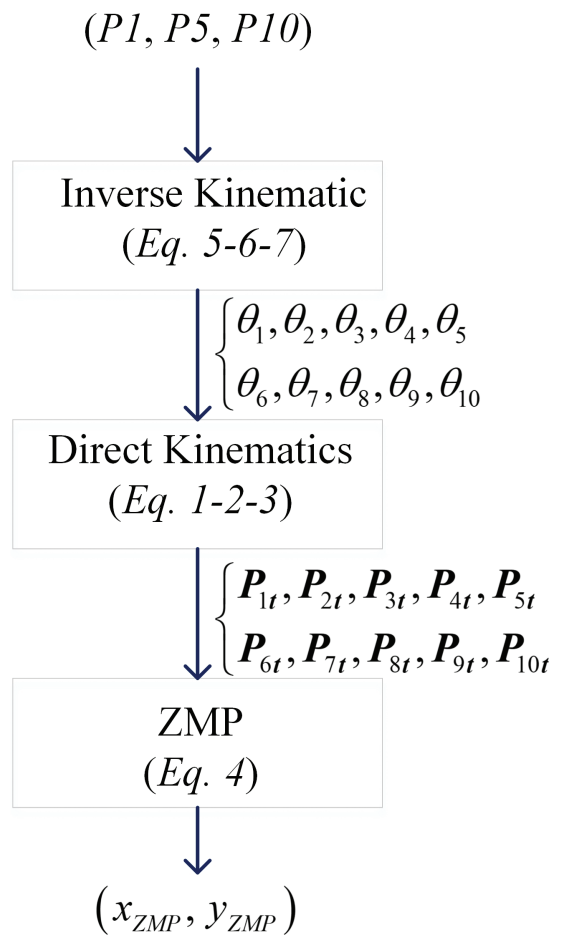

Figure 2 Calculation of the zero moment point. preset foot-lift magnitude, with the gait parameters of biped robot as variables. Specifically, we define the optimization problem as follows:

The goal of biped robot is to achieve a stable gait with preset footlift value. For this purpose, the ZMP point is to always be ensured within the foot area [6]. If the ZMP is within the area of the supporting leg, the robot does not fall [7]. The calculation of the ZMP of biped robots in walking is shown in Figure 2. The stability of the humanoid robot is quantified by the distance between the ZMP and the foot center in the step cycle. Walking gait with maximum stability is obtained by minimizing the function $\mathrm{f} 1$ in Equation (8) [1]:

$$
f_{1}=\int_{0}^{T} \sqrt{x_{Z M P}^{2}+y_{Z M P}^{2}} \cdot d t
$$

where $T$ denotes stepping cycle and $\left(x_{Z M P}, y_{Z M P}\right)$ denotes the coordination of ZMP point in the robot's process of stepping which is away from the quadrant in the center of the foot. In this paper the Equation (8) represents the first objective function.

Additionally, for the humanoid robot to follow the preset footlifting height value $-H_{r e f}$, the difference between the magnitude of the foot-lift value $H$ - and the foot-lift preset value $-H_{\text {ref }}$ (see Equation (9)) represents the second objective function.

$$
f_{2}=\left|H_{\text {ref }}-H\right|
$$

Thus, in order for biped robot to obtain a steady gait with the footlift set up in advance, we find the minimum value of the two objective functions $f_{1}$ and $f_{2}$, or similarly to find the minimum of the function $f$ as

$$
\left\{\begin{array}{l}
f=\lambda \cdot\left(\int_{0}^{T}\left(\sqrt{x_{Z M P}^{2}+y_{Z M P}^{2}}\right) \cdot d t\right)+(1-\lambda) \cdot\left|H_{r e f}-H\right| \\
-F_{x 1} \leq x_{Z M P} \leq F_{x 2} ;-F_{y 1} \leq y_{Z M P} \leq F_{y 2}
\end{array}\right.
$$

in which, $F_{x 1}+F_{x 2}$ and $F_{y 1}+F_{y 2}$ is the length and width of the biped robot foot, $\lambda(0 \leq \lambda \leq 1)$ can be used to satisfactorily select the priority between the walking stability ( $\lambda$ increase) and the variance with the desired foot-lifting magnitude ( $\lambda$ decreased).

To solve the problem, algorithm diagram of optimal walking gait for biped robots is shown in Figure 3. The $n$-WPG composes of the gait generator of the two-foot trajectory, the hip trajectory and the biped inverse kinematics (see additional works at [4]). The meta-heuristic optimization algorithms are used to create 4 gait parameters $(S, H$, $h, n)$ that provide the $n$-WPG in which outputs of the $n$-WPG are to create 10 angles for biped robot. The output of biped robot is the location of ZMP points as described in Figure 2. The optimal target function identified by the meta-heuristic optimization algorithm is a biped robot that walks steadily with the desired foot-lift, as presented in Equation (9). The following difference approximation is used to calculate the derivatives:

$$
\left\{\begin{array}{l}
\dot{x}_{i+1}=\frac{x_{i+1}-x_{i}}{\Delta t} ; \ddot{x}_{i+1}=\frac{\dot{x}_{i+1}-\dot{x}_{i}}{\Delta t} \\
\dot{y}_{i+1}=\frac{y_{i+1}-y_{i}}{\Delta t} ; \ddot{y}_{i+1}=\frac{\dot{y}_{i+1}-\dot{y}_{i}}{\Delta t} \\
\dot{z}_{i+1}=\frac{z_{i+1}-z_{i}}{\Delta t} ; \ddot{z}_{i+1}=\frac{\dot{z}_{i+1}-\dot{z}_{i}}{\Delta t}
\end{array}\right.
$$




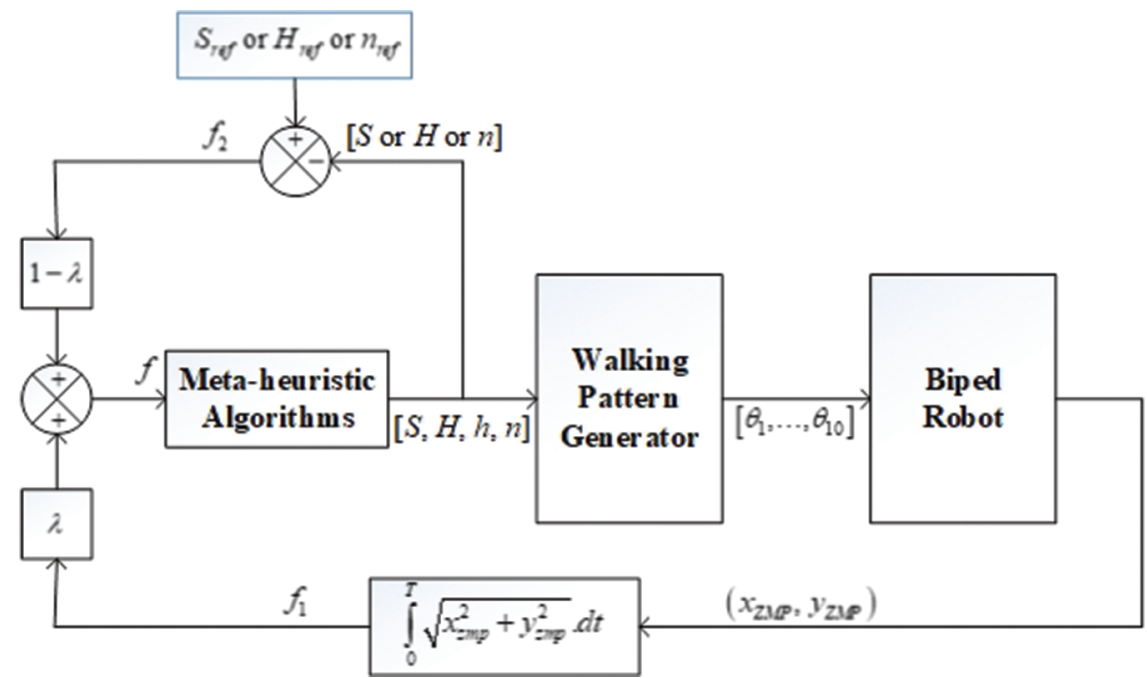

Figure 3 Algorithm diagram of optimal walking gait for biped robot.

In the simulation, $\left(\boldsymbol{P}_{1}, \boldsymbol{P}_{5}\right.$ and $\left.\boldsymbol{P}_{10}\right)$ are chosen intuitively. $\boldsymbol{P}_{1}$ and $\boldsymbol{P}_{10}$ are selected according to the desired leg movement for a specific activity. The choice of $\boldsymbol{P}_{5}$ depends on the mechanical structure of the biped as it involves shifting of ZMP from one foot to another. In a nature-walking sequence, which can be seen as combination of three sub-sequences: Starting step, Periodic steps and Ending step, $(0 \leq t \leq 3 \mathrm{~T}), \boldsymbol{P}_{1}$ and $\boldsymbol{P}_{10}$ for straight walking are provided by Equations (12) and (13) while $\boldsymbol{P}_{\mathbf{5}}$ for straight walking is given by Equation (14).

$$
\begin{cases}P_{1 x}(t)= \begin{cases}0 & , 0 \leq t<T \\ \frac{S}{2} \times\left(1-\sin \left(\frac{w}{2} t-\frac{\pi}{2}\right)\right) & , T \leq t \leq 2 T \\ S & , 2 T<t \leq 3 T\end{cases} \\ P_{1 y}(t)=0, \quad 0 \leq t \leq 3 T & , 0 \leq t<T \\ P_{1 z}(t)= \begin{cases}0 & , T \leq t \leq 2 T \\ H \times \sin \left(\frac{\pi}{2} \times\left(\sin \left(\frac{w}{2} t-\frac{\pi}{2}\right)+1\right)\right) & , 2 T<t \leq 3 T \\ 0 & , 2 T\end{cases} \end{cases}
$$

$$
\left\{\begin{array}{l}
P_{10 x}(t)= \begin{cases}\frac{S}{4} \times\left(1+\sin \left(\frac{w}{2} t-\frac{\pi}{2}\right)\right) & , 0 \leq t<T \\
\frac{S}{2} & , T \leq t \leq 2 T\end{cases} \\
P_{10 y}(t)=-w, 0 \leq t \leq 3 T \\
P_{10 z}(t)= \begin{cases}H \times \sin \left(\frac{\pi}{2} \times\left(\sin \left(\frac{w}{2} t-\frac{\pi}{2}\right)+1\right)\right), & 0 \leq t<T \\
0 \quad, \quad T \leq t \leq 2 T \\
H \times \sin \left(\frac{\pi}{2} \times\left(\sin \left(\frac{w}{2} t-\frac{5 \pi}{2}\right)+1\right)\right), & , 2 T<t \leq 3 T\end{cases}
\end{array}\right.
$$

$$
\left\{\begin{aligned}
& P_{5 x}(t)=\left\{\begin{array}{l}
\frac{S}{8} \times\left(1+\sin \left(\frac{w}{2} t-\frac{\pi}{2}\right)\right) \quad, 0 \leq t<T \\
\frac{S}{2} \times\left[1+\frac{1}{2} \sin \left(\frac{w}{2} t-\frac{3 \pi}{2}\right)\right], T \leq t \leq 2 T \\
\frac{S}{8} \times\left(7+\sin \left(\frac{w}{2} t-\frac{5 \pi}{2}\right)\right), 2 T<t \leq 3 T
\end{array}\right. \\
& P_{5 y}(t)=\left\{\begin{array}{c}
n \times \sin \left(\frac{\pi}{2} \times \sin \left(\frac{w}{4} t+1\right)\right) \times \sin \left(\frac{w}{2} t\right) \\
0 \leq t<T \\
n \times \sin \left(\frac{\pi}{2} \times\left(\sin \left(\frac{w}{4} t-\frac{\pi}{2}\right)+1\right)\right) \times \sin \left(\frac{w}{2} t\right) \\
T \leq t \leq 2 T \\
n \times \sin \left(\frac{\pi}{2} \times\left(1-\sin \left(\frac{w}{4} t\right)\right)\right) \times \sin \left(\frac{w}{2} t\right) \\
2 T<t \leq 3 T
\end{array}\right. \\
& P_{5 z}(t)=\left\{\begin{array}{c}
k_{1}+h \times \sin \left(\frac{\pi}{2} \times \sin \left(\frac{w}{4} t+1\right)\right) \times \sin \left(\frac{w}{2} t+\frac{\pi}{2}\right) \\
\quad 0 \leq t<T \\
k_{1}, \quad T \leq t \leq 2 T \\
k_{1}+h \times \sin \left(\frac{\pi}{2} \times \sin \left(\frac{w}{4}(3 T-t)+1\right)\right) \\
\quad \times \sin \left(\frac{w}{2}(3 T-t)+\frac{\pi}{2}\right), 2 T<t \leq 3 T
\end{array}\right.
\end{aligned}\right.
$$

in which, $T$ represents the time to perform a step of the humanoid robot and $k_{1}=d_{1}+d_{2}+d_{3}+d_{4}-h$.

The biped robot HUBOT-4 will walk stably with the preset footlift value. The coefficient $\lambda$ used in the objective function of Equation (10) is optimally selected equal 0.4 , and this $\lambda$ value will be used thorough the comparative testing process using GA, PSO, $\mathrm{CFO}, \mathrm{MDE}$ and proposed Jaya optimization algorithms. For optimal test based on GA, PSO, CFO, MDE and Jaya optimization algorithms, the four key gait parameters derived from the biped HUBOT- 4 are bounded as given in Table 2.

Simulation results are carried with MATLAB platform run with $2.49 \mathrm{GHz}$ CPU \& 8 GB RAM. Every method is tested 30 times. 
Table 3 eventually presents the GA, PSO, CFO, MDE and Jaya optimally selected parametric values.

Specify the foot-lifting height of biped HUBOT-4 being $H_{r e f}=2 \mathrm{~cm}$. Survey the performance of Jaya algorithm in optimal walking gait for biped robot by a full comparison to GA, PSO, CFO and MDE. The parameter of the algorithms is described in Table 3, selecting $\mathrm{NP}=32$ and GEN $=200$. Table 4 gives the comparative performance of the algorithms on the optimal procedure. Figure 4 presents the convergence rates of the algorithms using the average results of objective function in the optimal procedures. The comparative results in Table 4 show that the merit of Jaya algorithm has strongly better than GA, PSO, CFO and MDE.

The optimum set of 4 key parameters for biped HUBOT- 4 conformed to the objective derived from 30 runs per GA, PSO, CFO, MDE and proposed Jaya algorithms is shown in Table 5.

Based on the results described in Figure 4, it is important to notice that the Jaya algorithm searches for an optimal solution with an average value of 101.856724000000 after about 55 generations, while the MDE algorithm is approximately 134 generations after the search, finding an optimal solution obtained an average value of 101.856724300000, while the CFO algorithm must need around

Table 2 Bounds for four key walking gait parameters of humanoid HUBOT-4.

\begin{tabular}{lcc}
\hline Parameters & Lower Bound $(\mathbf{c m})$ & Upper Bound $(\mathbf{c m})$ \\
\hline$S$-Step length & 8 & 12 \\
$H$-foot lifting & 0.1 & 5 \\
$h$-kneeling & 1.1 & 1.5 \\
$n$-hip swinging & 0.1 & 6 \\
\hline
\end{tabular}

Table 3 Parameters of CFO, MDE and Jaya algorithm.

\begin{tabular}{lll}
\hline Method & Parameters & Value \\
\hline GA & Mutating rate & 0.2 \\
& Crossover rate & 0.7 \\
PSO & Accelerated factor 1 & 2 \\
& Accelerated factor 1 & 2 \\
& Inertia factor & 0.4 \\
CFO & Alpha & 0.25 \\
& Beta & 0.35 \\
& Gamma & 0.95 \\
& Frep & 0.5 \\
\multirow{2}{*}{ MDE } & deltaFrep & 0.05 \\
& Mutation value $(F)$ & Random $[0.4 ; 1.0]$ \\
Jaya & Crossover probability $(\mathrm{CR})$ & Random $[0.7 ; 1.0]$ \\
\hline
\end{tabular}

GA, Genetic Algorithm; PSO, Particle Swarm Optimization; CFO, Central Force Optimization; MDE, Modified Differential Evolution.
158 generations to find the optimal solution with an average value of 102.626134800000, while the PSO algorithm is approximately 41 generations after the search, finding an optimal solution obtained an average value of 101.885709700000 , while the GA algorithm must need around 190 generations to find the optimal solution with an average value of 102.291673400000. These results demonstrate that the Jaya algorithm outperforms GA, PSO, CFO and MDE in terms of convergence speed with the best target function value.

Table 5 shows the comparative optimized results of GA, PSO, CFO, $\mathrm{MDE}$ and proposed Jaya. The difference between the magnitude of the foot-lift value - $H$ and the foot-lift preset value $-H_{r e f}=2 \mathrm{~cm}$ of proposed Jaya proves better than GA, PSO and about - 0.5. Using comparative results tabulated in Table 5, it is evident to conclude that the proposed Jaya algorithm proves the best precise and robust optimization algorithm in comparison with the GA, PSO, MDE and CFO ones. Figure 5 shows the resulted ZMP trajectories when biped HUBOT- 4 steps along with a nature-walking sequence $(0 \leq t \leq 3 T)$ using GA, PSO, CFO, MDE and JAYA techniques.

The comparatively resulted ZMP trajectories using the five techniques illustrated in Figure 5 show that they surely keep in the supporting foot surface and it proves that biped allowing steadily robust stepping.

\section{CASE STUDIES}

The optimum identified solutions based on four principal biped WPG coefficients using proposed Jaya technique illustrated in Tables 6-8 which will strongly demonstrate that the eventual purpose of this study is completed with respect to the three following case studies which are investigated regarding to the preset foot-lift, the preset hip-shift and the preset step-length magnitudes, respectively.

\subsection{Case Study-1: Biped Optimal Stable Walking with Preset Foot-lift $\boldsymbol{H}_{\text {ref }}$ Magnitudes}

Table 6 demonstrates the optimized value of the walking gait parameters to ensure the biped HUBOT-4 to walk steadily with both cases corresponding to different preset foot-lift magnitudes. ( $H_{\text {ref }}=2 \mathrm{~cm}$ and $H_{\text {ref }}=3 \mathrm{~cm}$ ) optimized by Jaya algorithm. Figure 6 presents the convergence rates of the proposed Jaya algorithm using the average results of objective function in the optimal procedures.

Continually Figure $7 \mathrm{a}$ and $7 \mathrm{~b}$ illustrates the $2 \mathrm{D}$ gait in the $\mathrm{X}-\mathrm{Z}$ plane of the HUBOT-5, corresponding to two cases with different preset foot-lift amplitudes. The results from Table 6 and Figure 7 show

Table 4 Performance comparisons in optimal fitness function.

\begin{tabular}{lccccc}
\hline No & \multicolumn{2}{c}{ Optimal Objective Function } & \multirow{2}{*}{$\begin{array}{c}\text { Run } \\
\text { (Second) }\end{array}$} \\
\cline { 2 - 5 } & Best & Worst & Mean & Variance & \\
\hline Jaya & 101.856724029548 & $1.01856724 \mathrm{e}+02$ & $1.018567240000001 \mathrm{e}+02$ & $3.3425940701226 \mathrm{e}-27$ & 157 \\
MDE & 101.856724029550 & $1.01856724 \mathrm{e}+02$ & $1.018567260000000 \mathrm{e}+02$ & $3.5178160847427 \mathrm{e}-13$ & 153 \\
CFO & 101.889479300000 & $1.027262942 \mathrm{e}+02$ & $1.026317738400000 \mathrm{e}+02$ & 0.606345370124322 & 159 \\
PSO & 101.856724029549 & $1.027262942 \mathrm{e}+02$ & $1.018857096733334 \mathrm{e}+02$ & 0.025205077757601 & 152 \\
GA & 101.927584300000 & $1.030504002 \mathrm{e}+02$ & $1.022916734133333 \mathrm{e}+02$ & 0.057276108848447 & 158 \\
\hline
\end{tabular}

GA, Genetic Algorithm; PSO, Particle Swarm Optimization; CFO, Central Force Optimization; MDE, Modified Differential Evolution. 


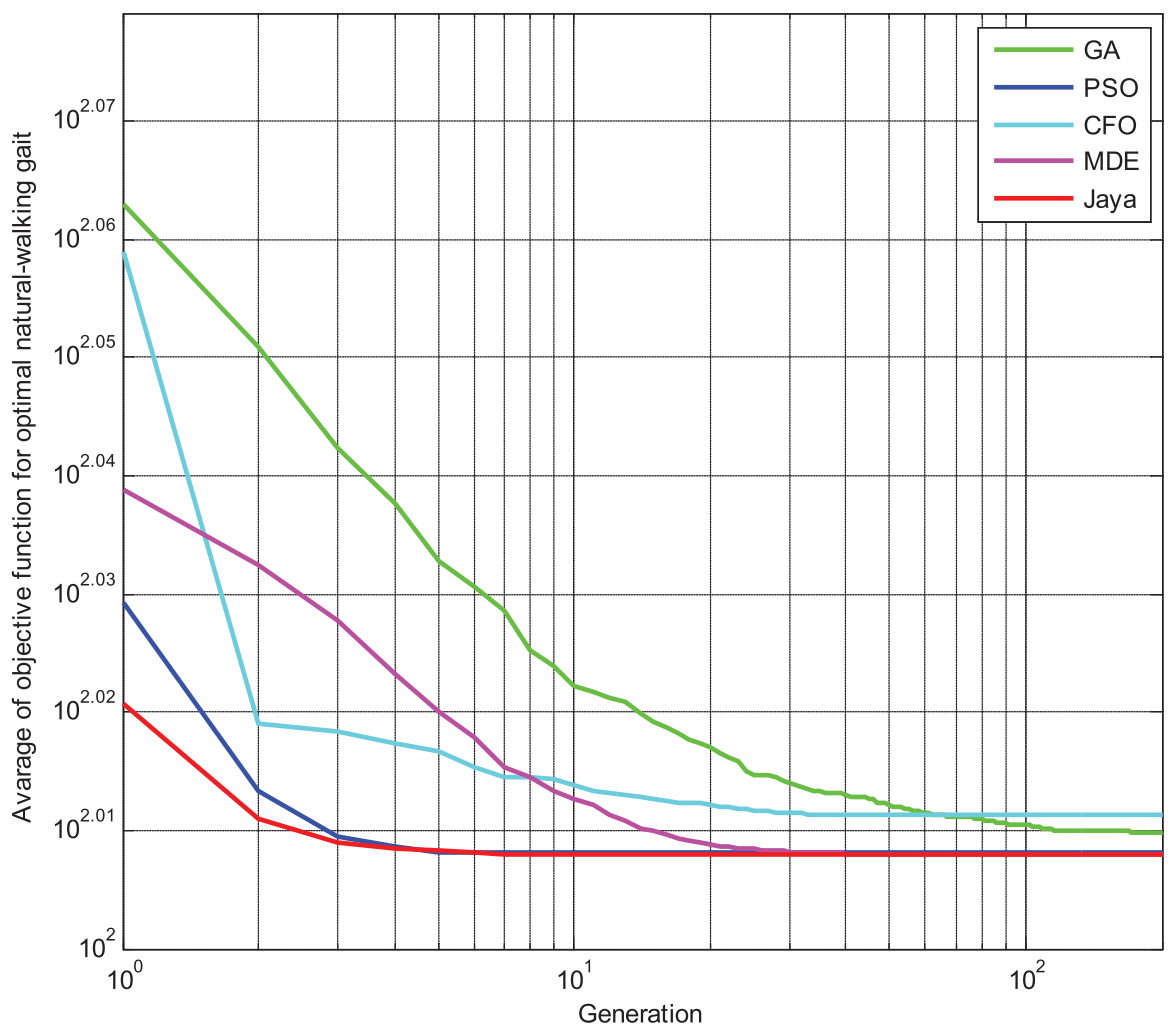

Figure 4 Convergence rate of the comparative algorithms in the optimal process.

Table 5 Resulted parametric set for five comparative algorithms.

\begin{tabular}{lccccc}
\hline & \multicolumn{5}{c}{$\boldsymbol{H}_{\boldsymbol{r e f}}=\mathbf{2} \mathbf{~ c m}$} \\
\hline Algorithms & \multicolumn{3}{c}{ Walking Gait Parameters Value } & $\begin{array}{c}\text { Best Fitness Value } \\
\boldsymbol{f}(\mathbf{c m})\end{array}$ \\
\cline { 2 - 5 } & $\boldsymbol{S}(\mathbf{c m})$ & $\boldsymbol{H}(\mathbf{c m})$ & $\boldsymbol{h}(\mathbf{c m})$ & $\boldsymbol{n}(\mathbf{c m})$ & \\
\hline GA & 11.99 & 1.99 & 1.1 & 5.97 & 101.927584300000 \\
PSO & 12.00 & 1.99 & 1.1 & 6.00 & 101.856724029549 \\
CFO & 11.97 & 1.99 & 1.1 & 5.97 & 101.889479300000 \\
MDE & 12.00 & 2.00 & 1.1 & 5.99 & 101.856724029550 \\
Jaya & 12.00 & 2.00 & 1.1 & 6.00 & 101.856724029548 \\
\hline
\end{tabular}

GA, Genetic Algorithm; PSO, Particle Swarm Optimization; CFO, Central Force Optimization; MDE, Modified Differential Evolution.

Table 6 Optimal parameter set.

\begin{tabular}{lcccc}
\hline $\boldsymbol{H}_{\boldsymbol{r e f}}(\mathrm{cm})$ & \multicolumn{4}{c}{ Jaya Optimization Results } \\
\cline { 2 - 5 } & $\boldsymbol{S}(\mathbf{c m})$ & $\boldsymbol{H}(\mathbf{c m})$ & $\boldsymbol{h}(\mathbf{c m})$ & $\boldsymbol{n}(\mathbf{c m})$ \\
\hline $\mathbf{2 . 0}$ & 12.0000 & 2.0000 & 1.1000 & 6.0000 \\
$\mathbf{3 . 0}$ & 12.0000 & 3.0000 & 1.1000 & 6.0000 \\
\hline
\end{tabular}

The bold values represent the reference values based on them to optimize the parameter set using Jaya.

Table 7 Optimal parameter set.

\begin{tabular}{lcccc}
\hline$n_{r e f}(\mathrm{~cm})$ & \multicolumn{4}{c}{ Jaya Optimization Results } \\
\cline { 2 - 5 } & $\boldsymbol{S}(\mathbf{c m})$ & $\boldsymbol{H}(\mathbf{c m})$ & $\boldsymbol{h}(\mathbf{c m})$ & $\boldsymbol{n}(\mathbf{c m})$ \\
\hline $\mathbf{4 . 0}$ & 12.0000 & 1.5000 & 1.1000 & 4.0000 \\
$\mathbf{5 . 0}$ & 12.0000 & 1.5000 & 1.1000 & 5.0000 \\
\hline
\end{tabular}

The bold values represent the reference values based on them to optimize the parameter set using Jaya.

that the biped HUBOT-4 attains a pickup lift in term of the preset foot-lift value.

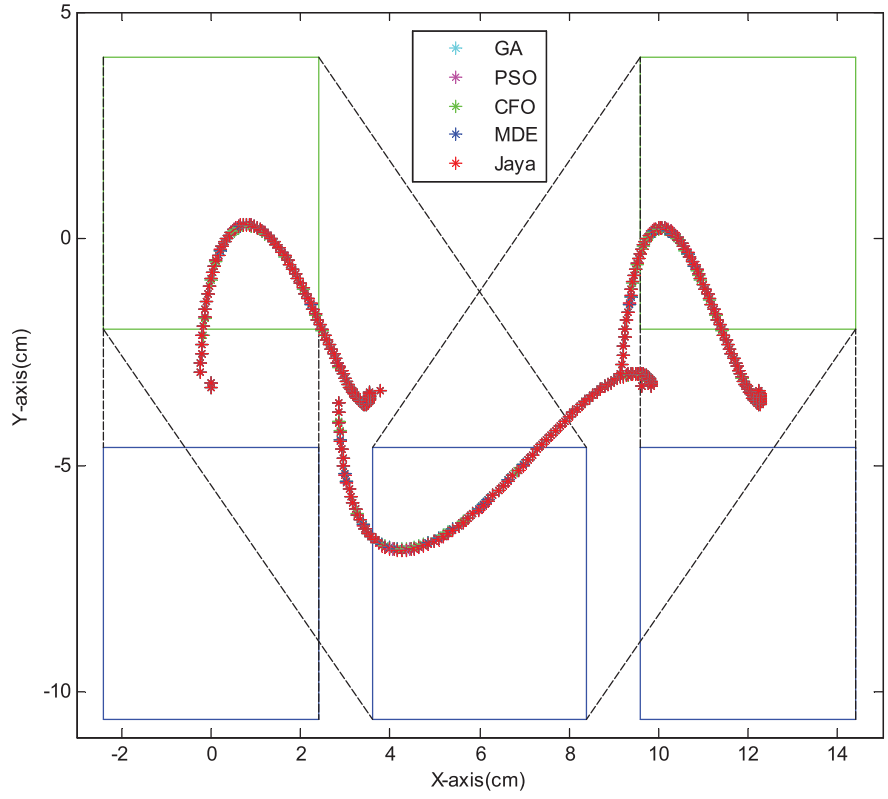

Figure 5 Resulted comparative Zero Moment Point (ZMP) survey.

Table 8 Optimal parameter set.

\begin{tabular}{lrccc}
\hline$S_{\text {ref }}(\mathrm{cm})$ & \multicolumn{4}{c}{ Jaya Optimization Results } \\
\cline { 2 - 5 } & $\boldsymbol{S}(\mathrm{cm})$ & $\boldsymbol{H}(\mathrm{cm})$ & $\boldsymbol{h}(\mathrm{cm})$ & $\boldsymbol{n}(\mathrm{cm})$ \\
\hline $\mathbf{9 . 0}$ & 9.0000 & 2.0000 & 1.1000 & 6.0000 \\
$\mathbf{1 0}$ & 10.0000 & 1.0000 & 1.1000 & 6.0000
\end{tabular}

The bold values represent the reference values based on them to optimize the parameter set using Jaya. 


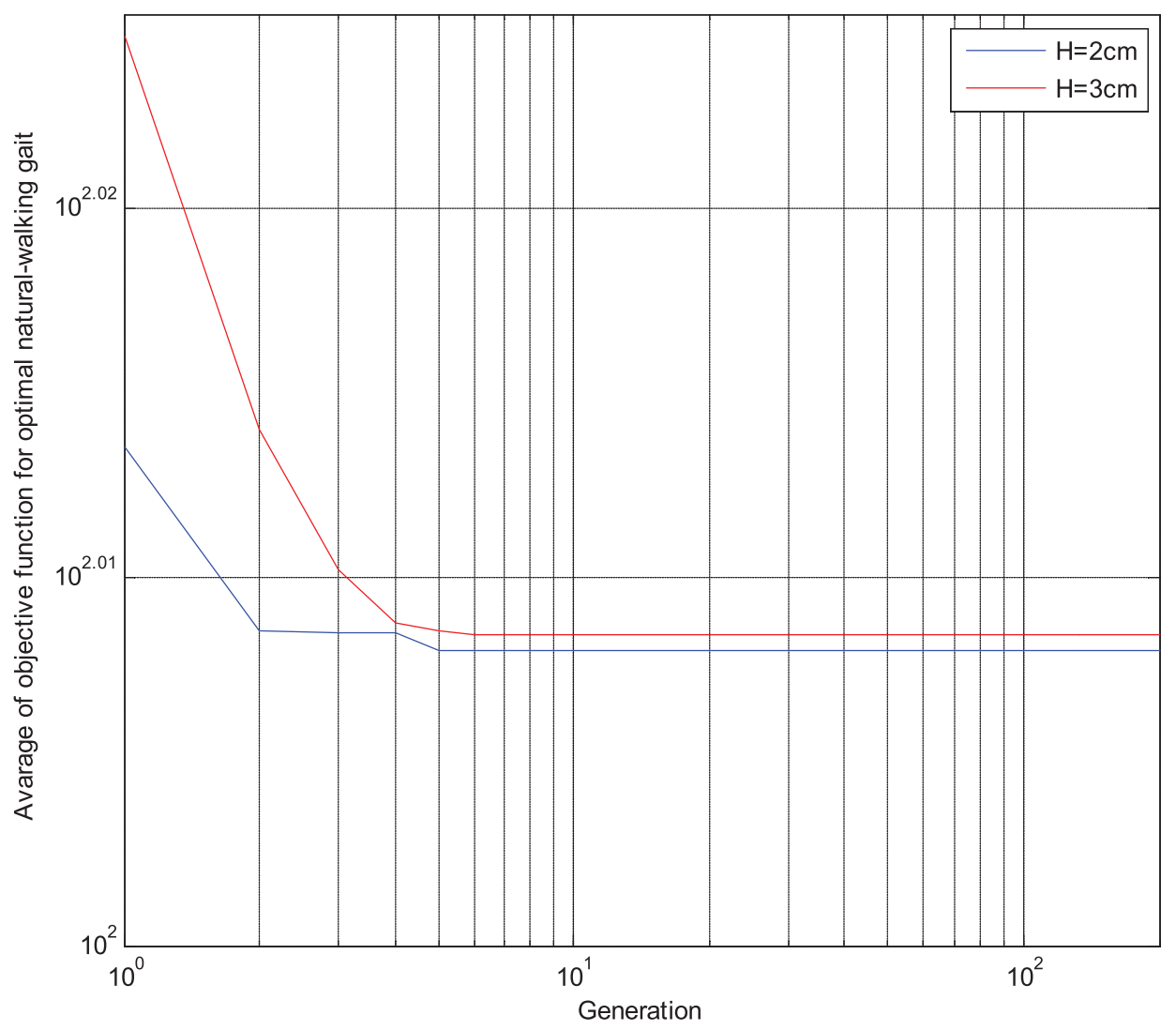

Figure 6 Convergence rate of the proposed Jaya algorithm in the optimal process.
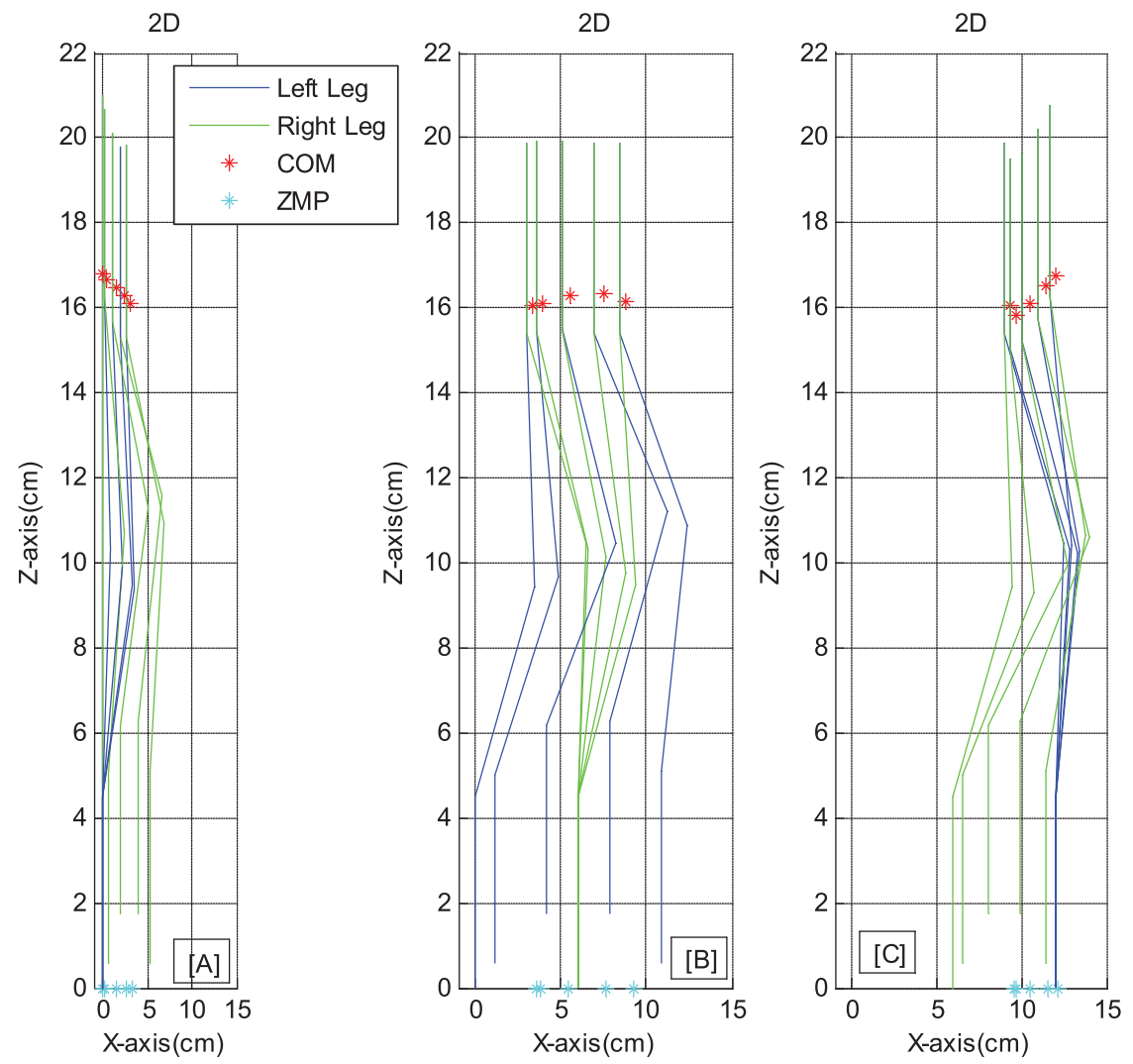

Figure 7a Stick diagram of biped robot for a nature-walking sequence in the $\mathrm{x}-\mathrm{z}$ plan. [A]: Starting step. [B]: Periodic steps. [C]: Ending step. 

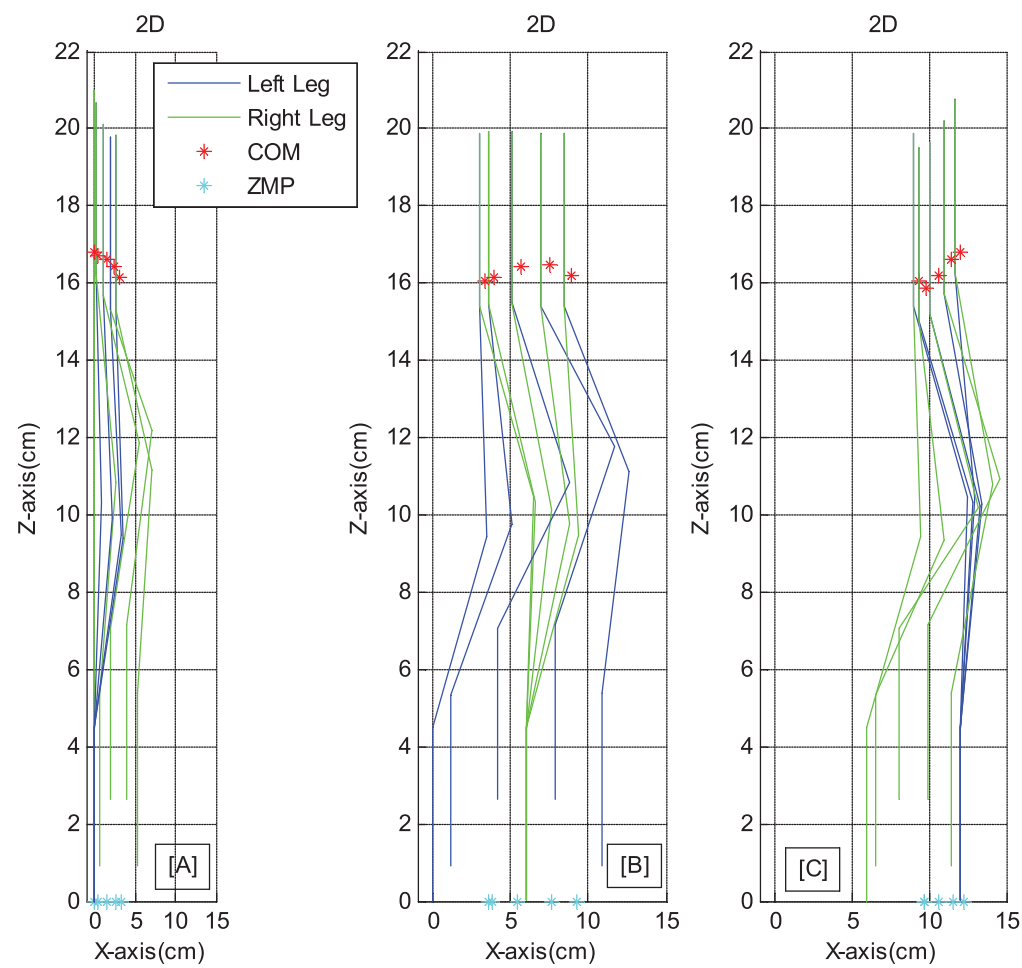

Figure $\mathbf{7 b}$ Stick diagram of biped robot for a nature-walking sequence in the $\mathrm{x}-\mathrm{z}$ plan. [A]: Starting step. [B]: Periodic steps. [C]: Ending step.

Furthermore Figure $8 \mathrm{a}$ and $8 \mathrm{~b}$ illustrates the resulted ZMP point trajectory and the projection of Groundd-Center-Of-Mass (GCOM) trajectory for two different preset foot-lifting amplitudes. This result shows that the ZMP point is always in the supporting foot area and then it ensures that the HUBOT-4 biped robot surely keeps stable walking.

Figure 9 below demonstrates the 10 rotary angular trajectories in one stepping cycle of the two legs of biped HUBOT-4 $\left(\theta_{1}, \theta_{2}, \theta_{3}, \theta_{4}, \theta_{5}, \theta_{6}, \theta_{7}, \theta_{8}, \theta_{9}, \theta_{10}\right)$ during walking with two optimally resulted sets of gait parameters with respect to two different preset foot-lift values (tabulated in Table 6).

Based on the results of the optimization and simulation shown in Table 6, Figures 7-9, which convincingly demonstrate that the work of preset foot-lift parameter - $H_{r e f}$ and four optimally selected parameters ( $S$-step length, $H$-foot lifting, $h$ - kneeling and $n$ - hip swinging) ensuring the HUBOT-4 biped robot to steadily walking without falling apart and keeping pace with desired foot-lift amplitude. The proposed algorithm with gait parameters optimized by Jaya algorithm is convincingly feasible.

\subsection{Case Study-2: Biped Optimal Stable Walking with Preset Hip-Shift $n_{\text {ref }}$ Values}

Table 7 demonstrates the optimum identified value of proposed WPG to guarantee the biped robot to step stably in two different cases regarding to various preset hip-shift values $\left(n_{\text {ref }}=4 \mathrm{~cm}\right.$ and $n_{\text {ref }}=5 \mathrm{~cm}$ ) optimized by Jaya algorithm, and Figure 10 presents the convergence rate optimized by the proposed Jaya algorithm using the average results of objective function in the optimal procedures.
Furthermore Figure $11 \mathrm{a}$ and $11 \mathrm{~b}$ below presents the resulted 2D gait in the $Y-Z$ co-ordinate of the biped, with respect to three different cases of preset hip-shift values. Results from Table 7 and Figure 11 strongly ensure that the humanoid robot successfully obtains a desired hip-shift value in control process.

Figure 12 illustrates the resulted ZMP values for two preset hip-shift magnitudes. These results confirm that ZMP trajectory is always to be kept inside the supporting footprint surface and thus guarantees humanoid robot walking efficiently and robustly.

Figure 13 demonstrates the 10 rotary angular trajectories in one stepping cycle of the two legs of biped HUBOT-4 $\left(\theta_{1}, \theta_{2}, \theta_{3}, \theta_{4}, \theta_{5}, \theta_{6}, \theta_{7}, \theta_{8}, \theta_{9}, \theta_{10}\right)$ during walking with two optimally resulted sets of gait parameters with respect to two different preset hip-shift values and tabulated in Table 8.

In summary based on the results of the optimization and simulation shown in Table 7, Figures 11-13, which once more demonstrate that the work of preset hip-shift parameter - $n_{\text {ref }}$ and four optimally selected parameters ( $S$-step length, $H$-foot lifting, $h$ - kneeling and $n$ - hip swinging) ensuring the HUBOT-4 biped robot to steadily walking without falling apart and keeping pace with desired hipshift amplitude. The proposed algorithm with gait parameters optimized by Jaya algorithm is convincingly feasible.

\subsection{Case Study-3: Biped Optimal Stable Walking with Preset Step-Length $S_{\text {ref }}$ Values}

Table 8 demonstrates the optimum identified value of proposed WPG to guarantee the humanoid robot to step stably in two 


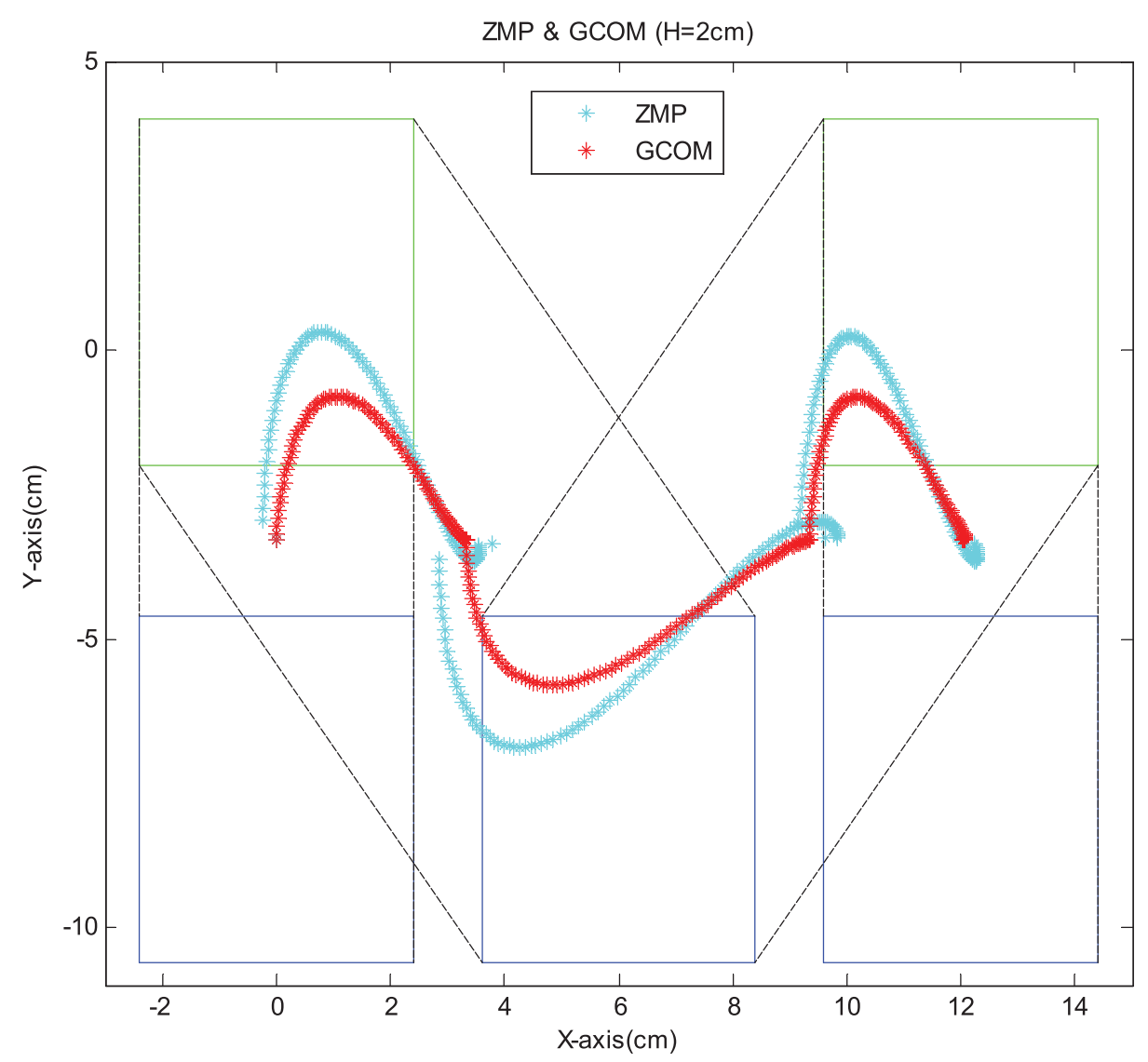

Figure 8a $\quad$ Resulted Zero Moment Point (ZMP) và Grounded-Center-Of-Mass (GCOM) trajectories.

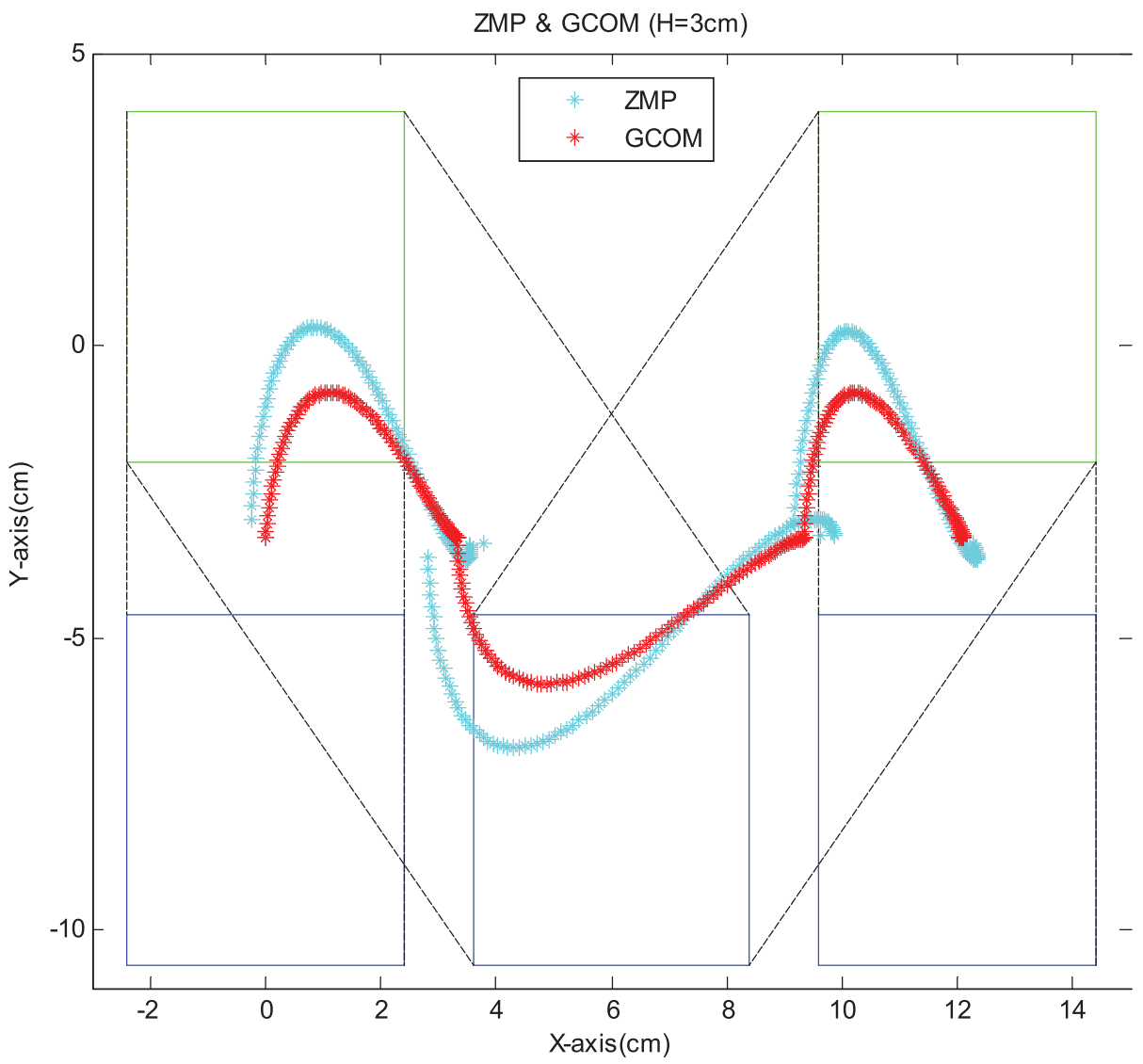

Figure 8b Resulted Zero Moment Point (ZMP) và Grounded-Center-Of-Mass (GCOM) trajectories. 

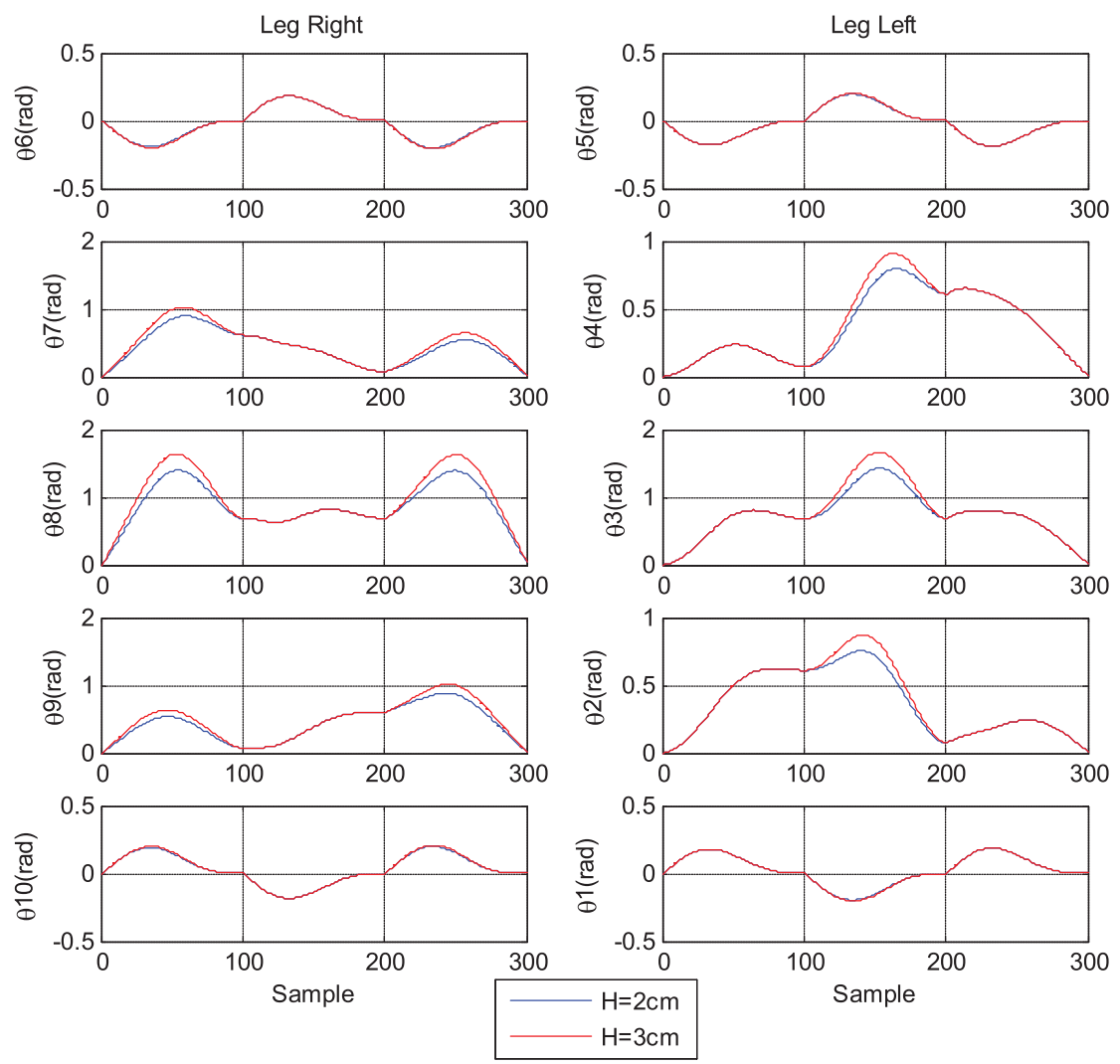

Figure 9 Trajectories of the ten joint angles located at two legs of biped HUBOT-4.

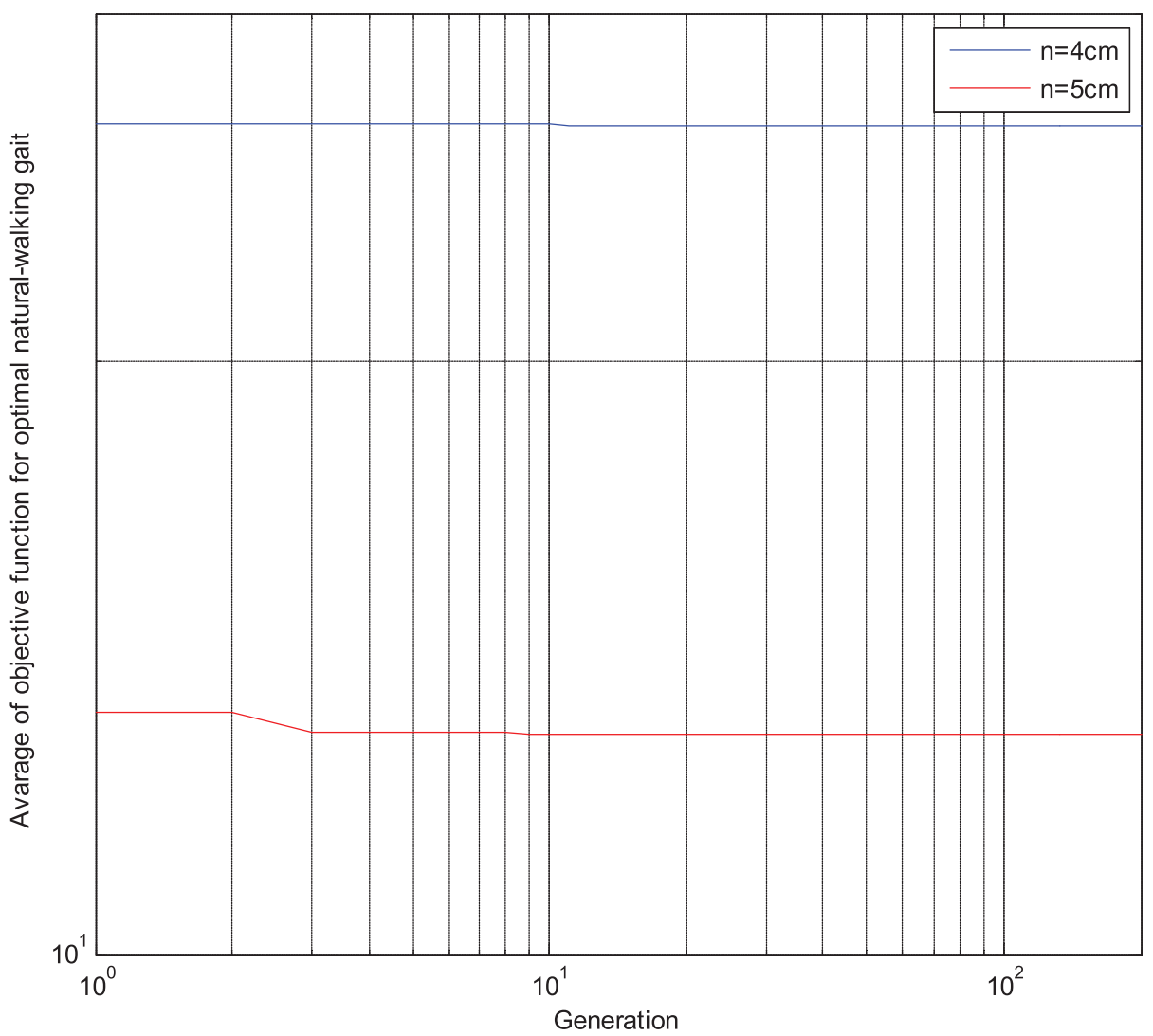

Figure 10 Convergence rate using the proposed Jaya algorithm in the optimal procedure. 

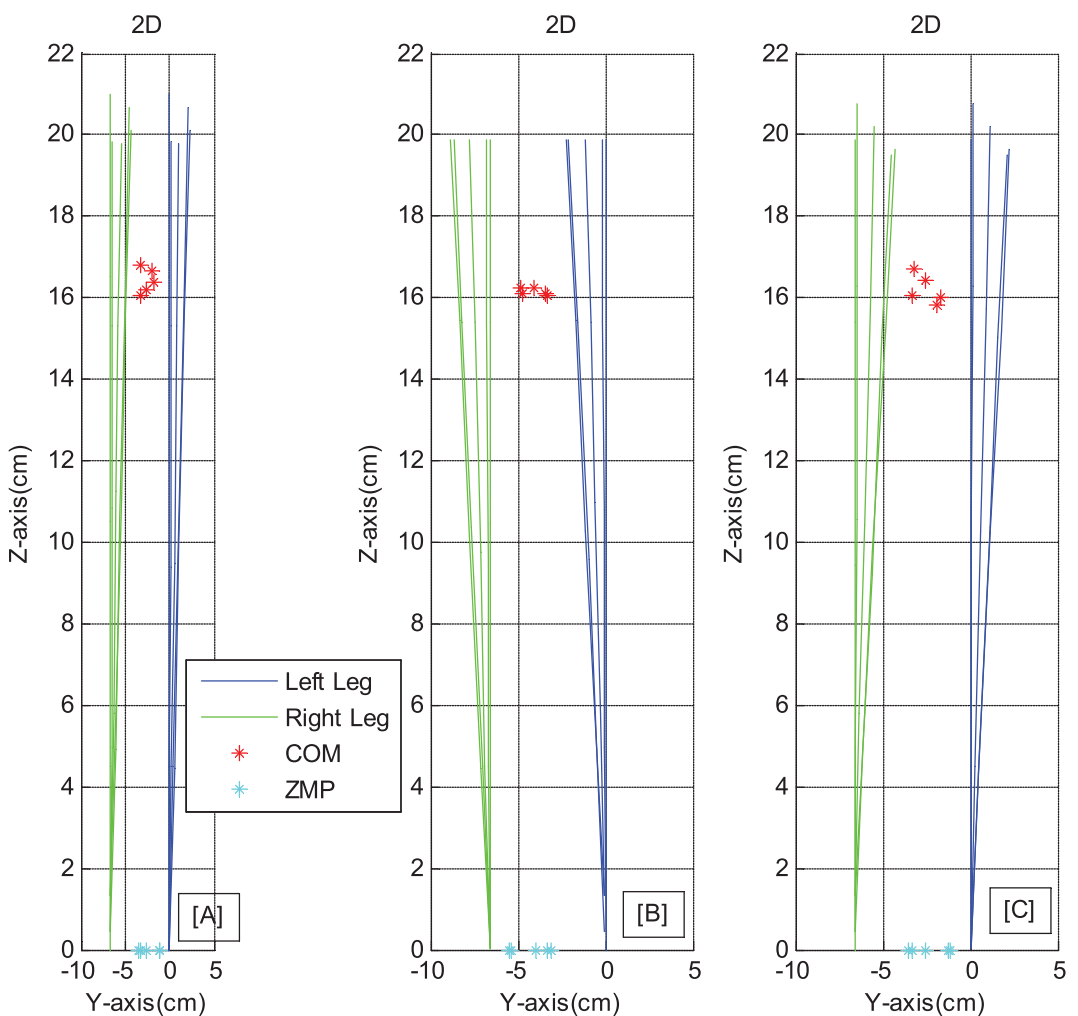

Figure 11a $\mid$ Resulted 2D gait of humanoid robot with $n_{\text {ref }}=4 \mathrm{~cm}$. [A]: Starting step. [B]: Periodic steps. [C]: Ending step.
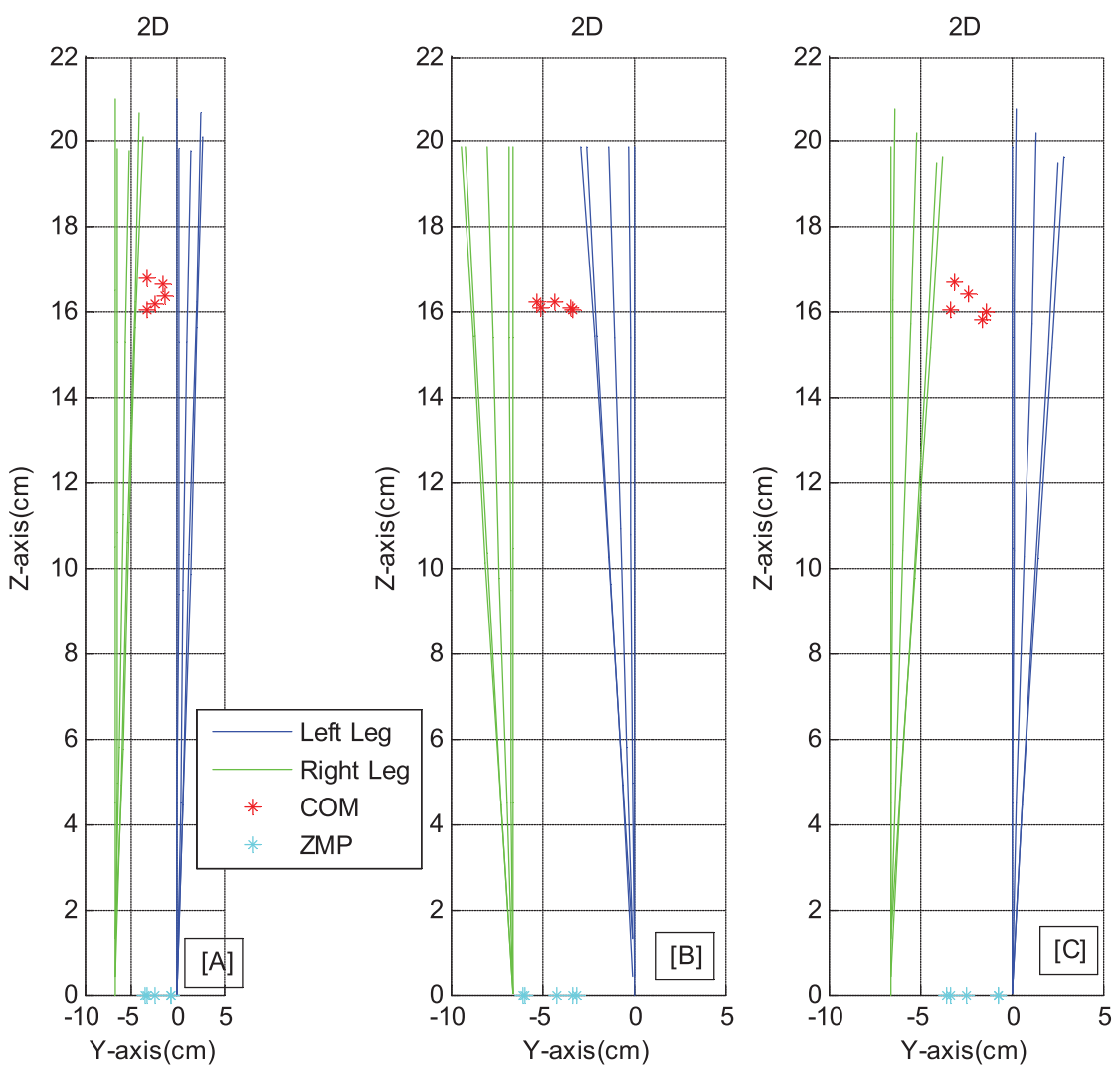

Figure 11b Resulted 2D gait of humanoid robot with $n_{\text {ref }}=5 \mathrm{~cm}$. [A]: Starting step.

[B]: Periodic steps. [C]: Ending step. 


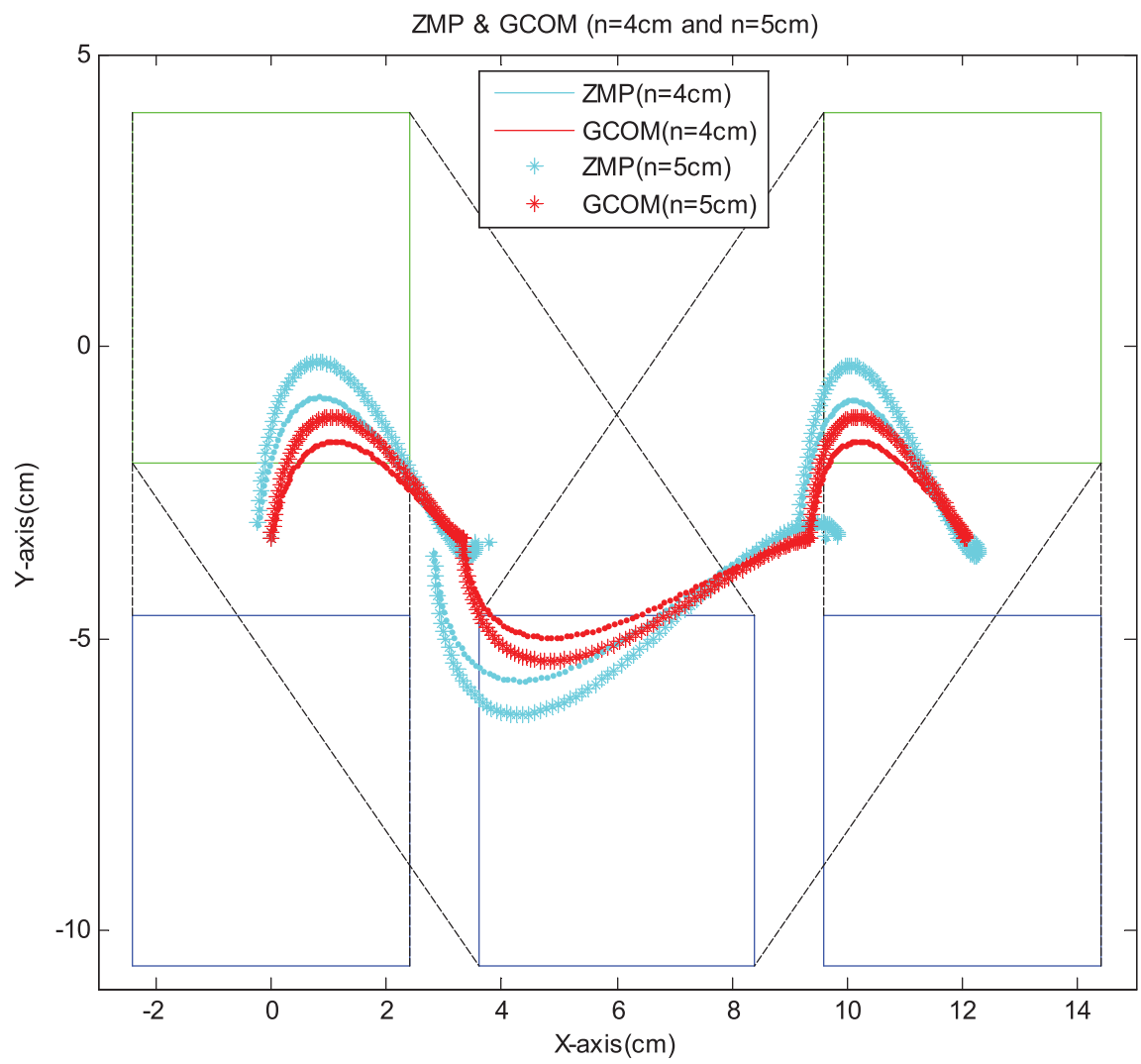

Figure $12 \mid$ Resulted Zero Moment Point (ZMP) trajectories.
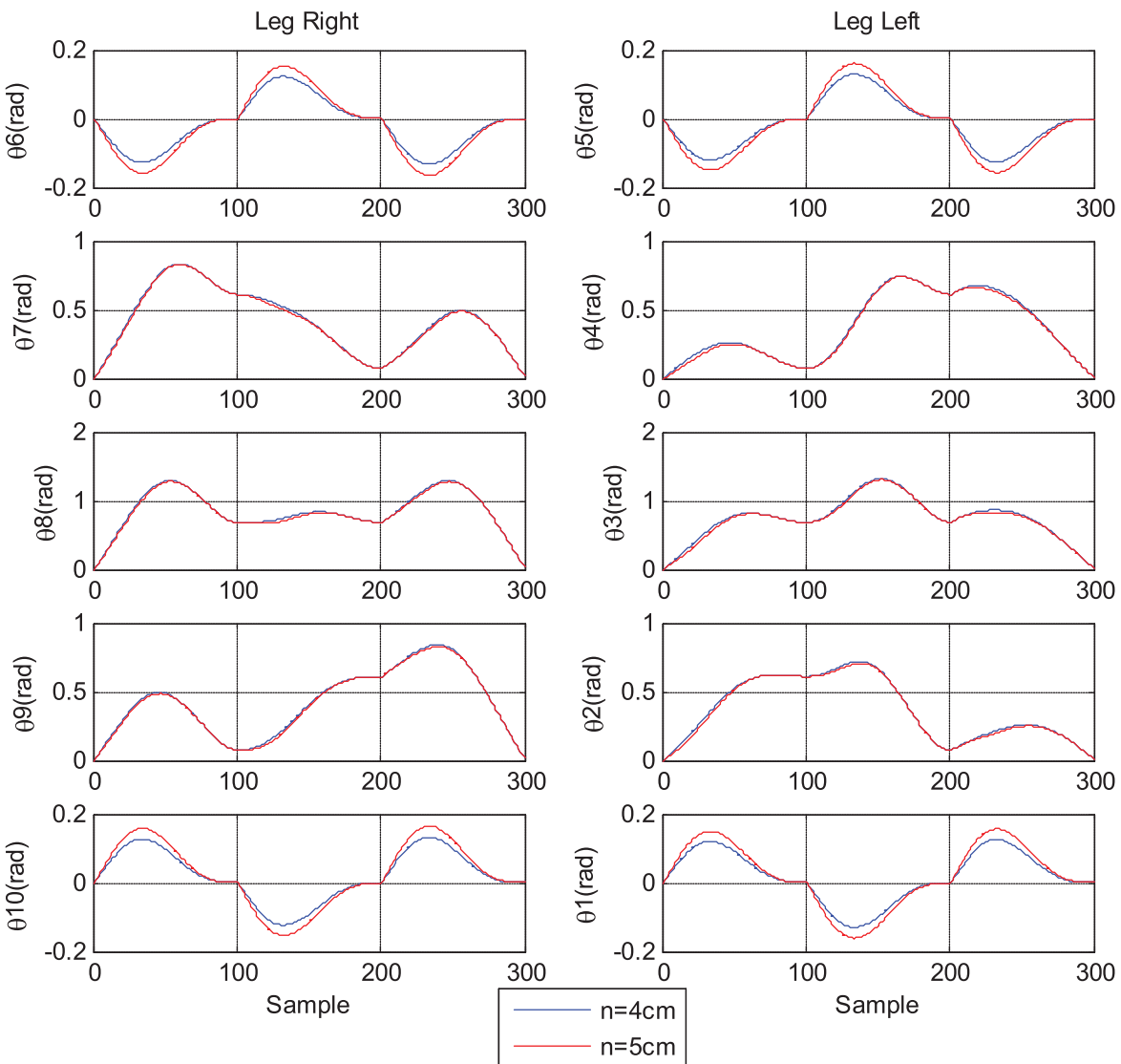

Figure 13 Trajectories of the ten joint angles located at two legs of biped HUBOT-4. 


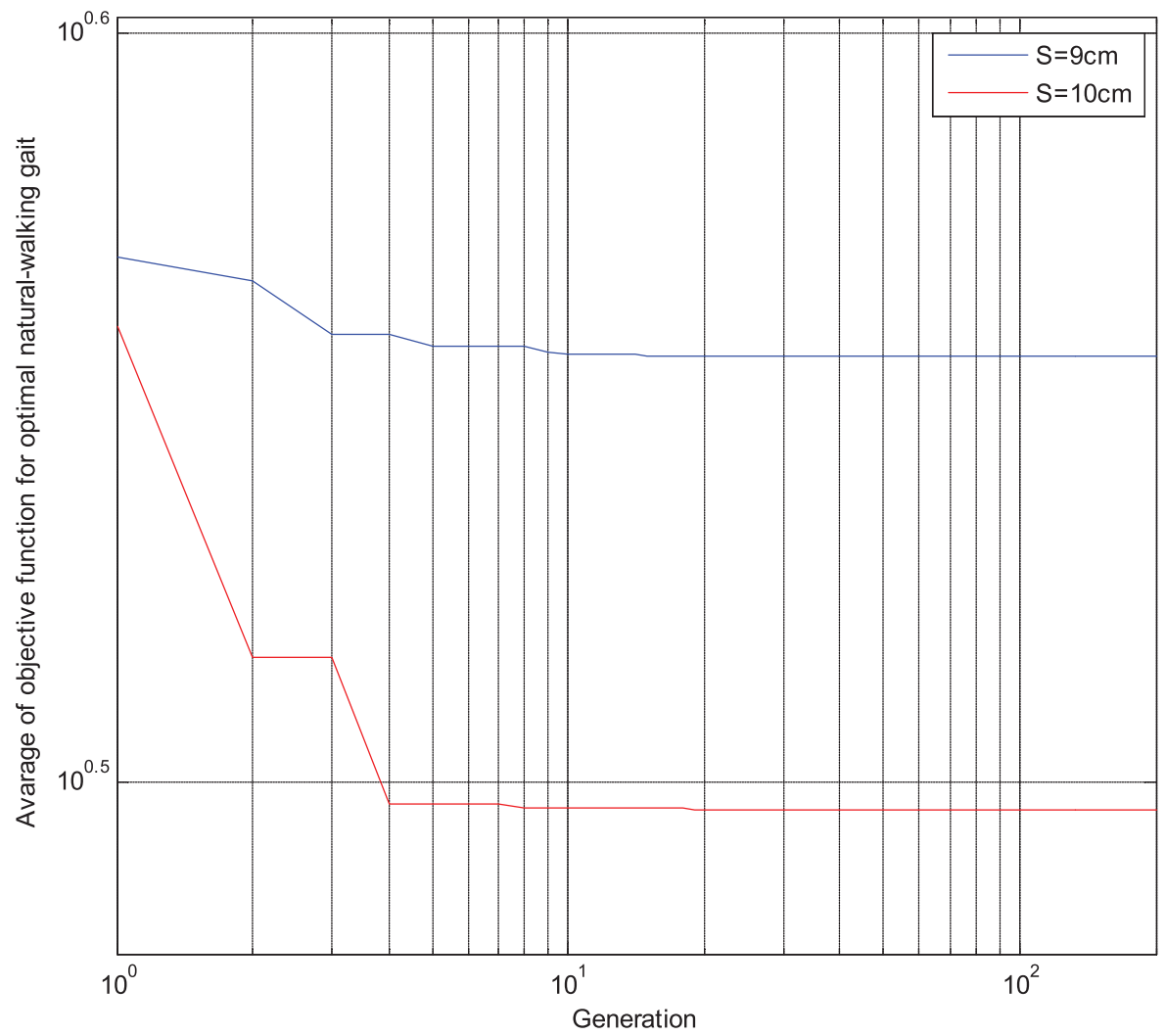

Figure 14 Convergence rates optimized with proposed Jaya algorithm in the optimal procedure.

different cases regarding to various preset step-length values $\left(S_{\text {ref }}=\right.$ $9 \mathrm{~cm}$ and $S_{\text {ref }}=10 \mathrm{~cm}$ ) optimized by Jaya algorithm, and Figure 14 presents the convergence rates using the proposed Jaya algorithm based on the average results of objective function in the optimal procedures.

Continually Figure $15 \mathrm{a}$ and $15 \mathrm{~b}$ presents the resulted $2 \mathrm{D}$ gait in the $\mathrm{X}-\mathrm{Z}$ co-ordinate of the biped, with respect to different cases of preset step-length values. Results from Table 8 and Figure 15 strongly ensure that the biped robot successfully obtains a desired step-length value in control process.

Then Figure 16a and 16b illustrates the resulted ZMP values for two preset step-length magnitudes. These results confirm that ZMP trajectory keeps inside the supporting footprint surface and thus guarantees humanoid robot walking efficiently and robustly.

Consequently results from Figure 17 demonstrate the 10 rotary angular trajectories in one stepping cycle of the two legs of biped HUBOT-4 $\left(\theta_{1}, \theta_{2}, \theta_{3}, \theta_{4}, \theta_{5}, \theta_{6}, \theta_{7}, \theta_{8}, \theta_{9}, \theta_{10}\right)$ during walking with two optimally resulted sets of gait parameters with respect to two different preset step-length values and tabulated in Table 8 .

In summary based on the results of the optimization and simulation shown in Table 8, Figures 15-17, which once more strongly demonstrate that the work of preset step-length parameter - $S_{\text {ref }}$ with four optimally selected parameters $(S$-step length, $H$-foot lifting, $h$ - kneeling and $n$ - hip swinging) ensuring the HUBOT-4 biped robot to steadily walking without falling apart and keeping pace with desired step-length amplitude. The proposed algorithm with gait parameters optimized by Jaya algorithm proves convincingly feasible.

\section{CONCLUSIONS}

In this paper the optimization of the biped walking gait trajectory available to stably and naturally walking with preset steplength magnitude, has been obtained by quantifying the distance between the ZMP and the foot center in the step cycle and optimally quantifying the difference between the magnitude of steplength value and the step-length preset one. We applied Jaya optimization algorithm to minimize them by treating the gait parameters of biped robot as variables. This innovative concept has been verified by a computer simulation of a real biped robot HUBOT-4. Since the optimization process is performed for each time interval, the proposed technique can generate the biped gait trajectory in real-time.

\section{CONFLICT OF INTEREST}

Authors confirm that there is no any conflict of interest in this paper. 

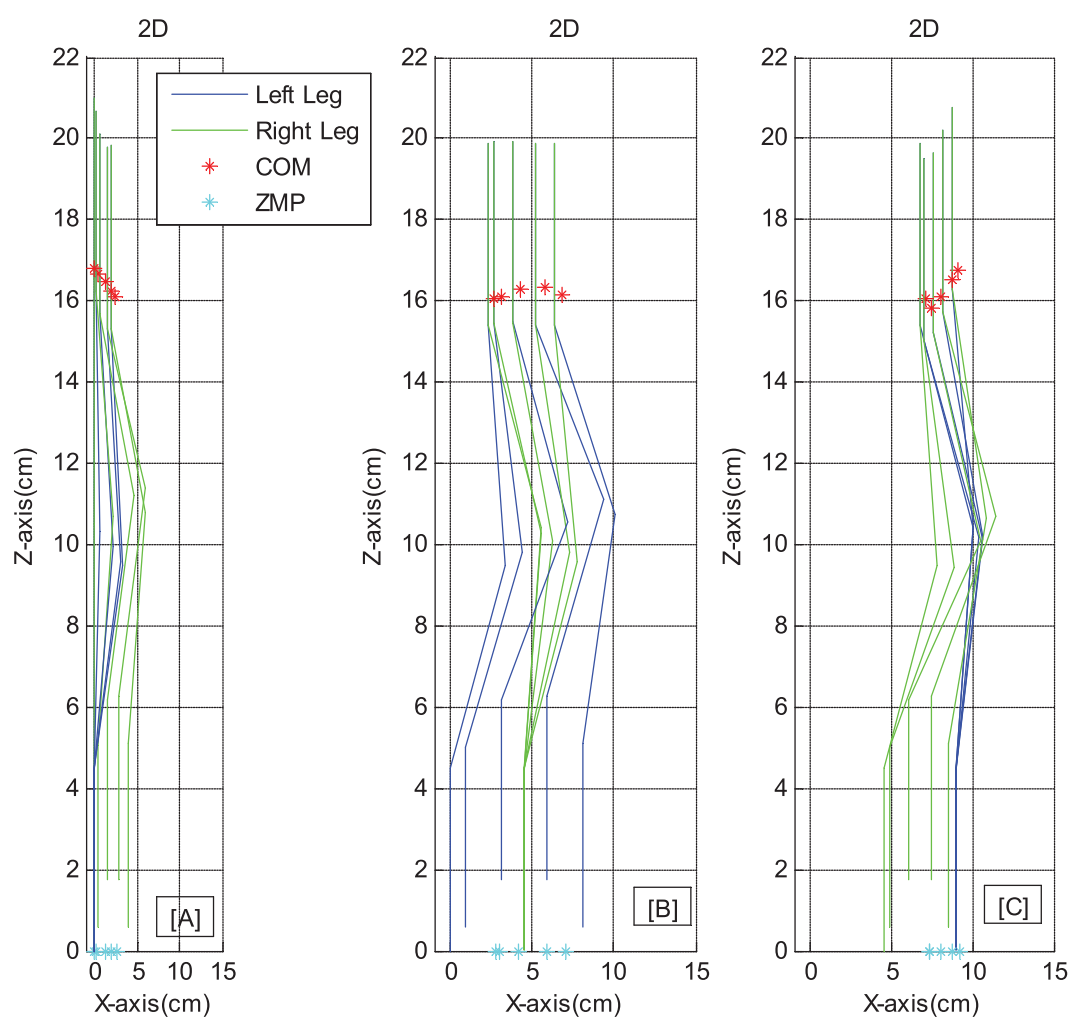

Figure 15a $\quad$ Resulted 2D gait of biped robot with step-length $S_{\text {ref }}=9 \mathrm{~cm}$. [A]: Starting step. [B]: Periodic steps. [C]: Ending step.
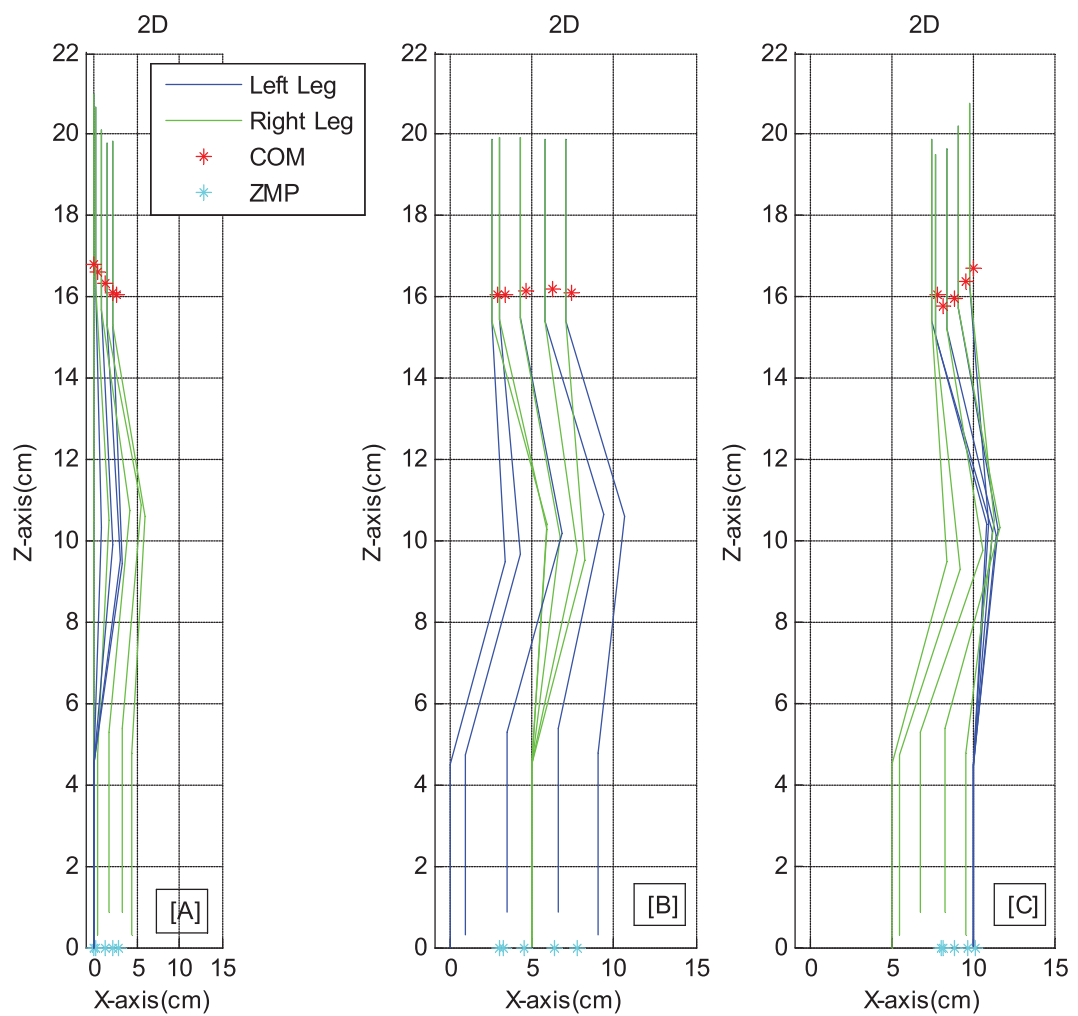

Figure 15b Resulted 2D gait of biped robot with $S_{\text {ref }}=10 \mathrm{~cm}$. [A]: Starting step. [B]: Periodic steps. [C]: Ending step. 


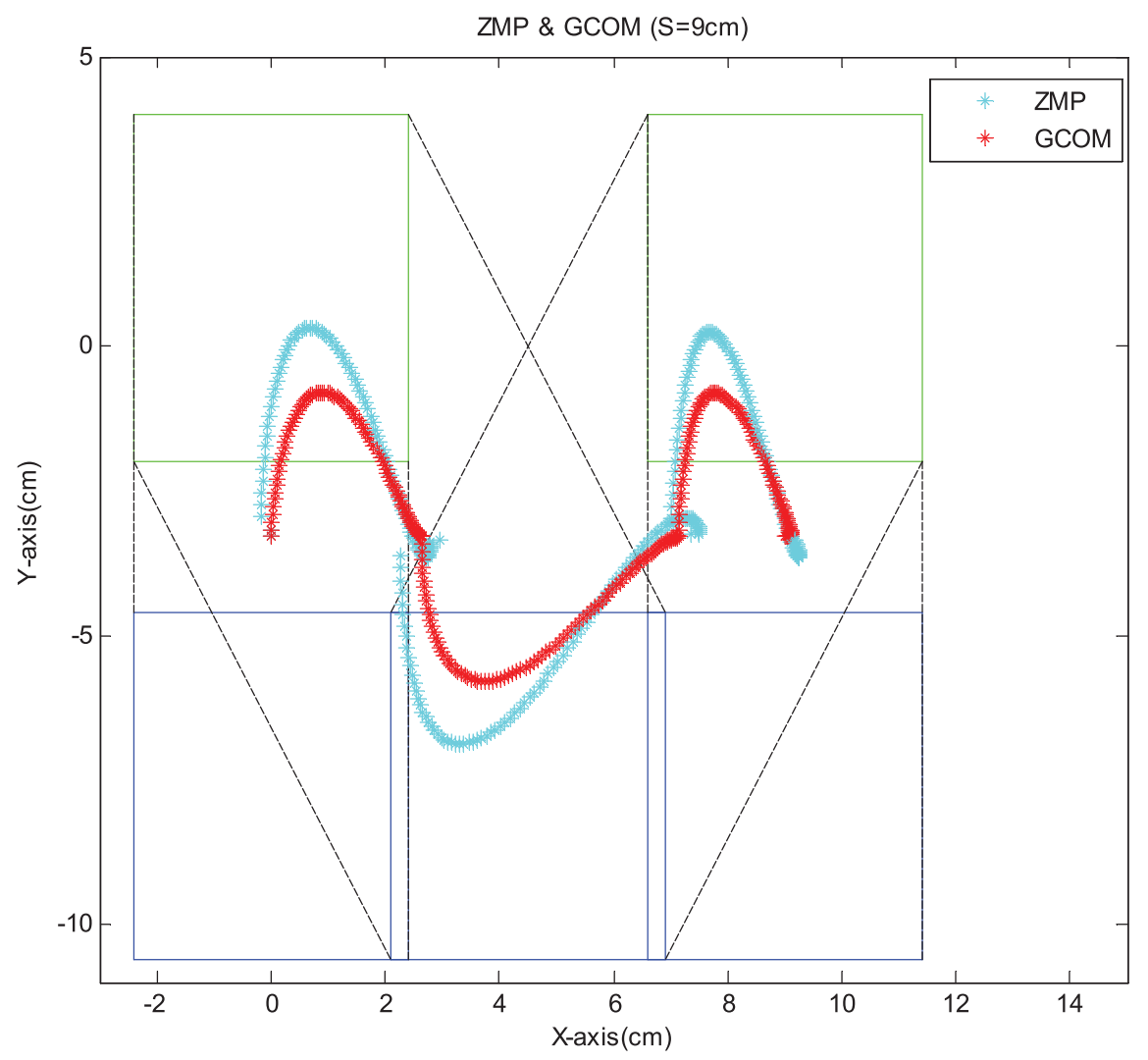

Figure 16a $\mid$ Resulted Zero Moment Point $(\mathrm{ZMP})$ trajectories with $S_{r e f}=9 \mathrm{~cm}$.

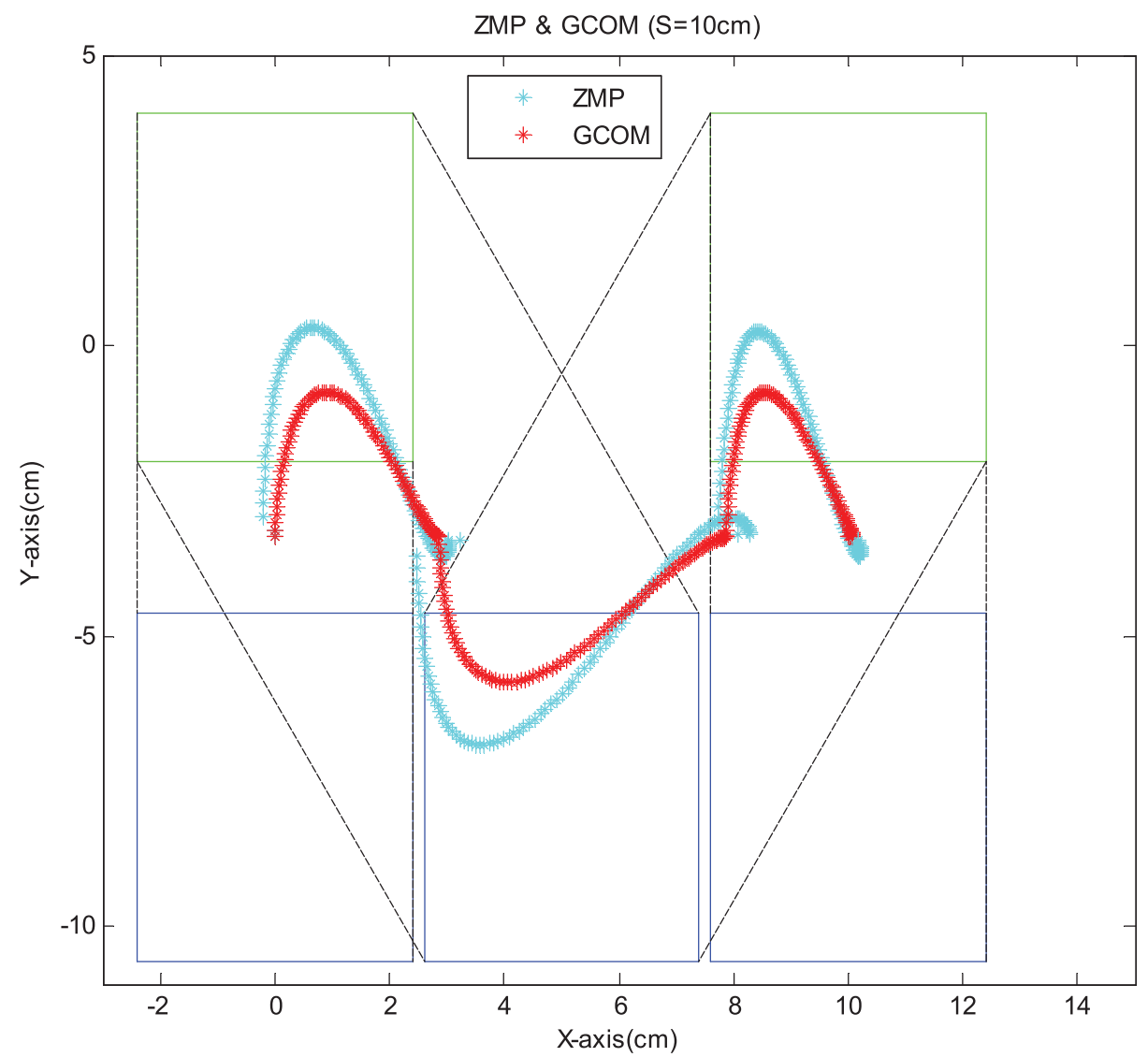

Figure 16b Resulted Zero Moment Point (ZMP) trajectories with $S_{\text {ref }}=10 \mathrm{~cm}$. 

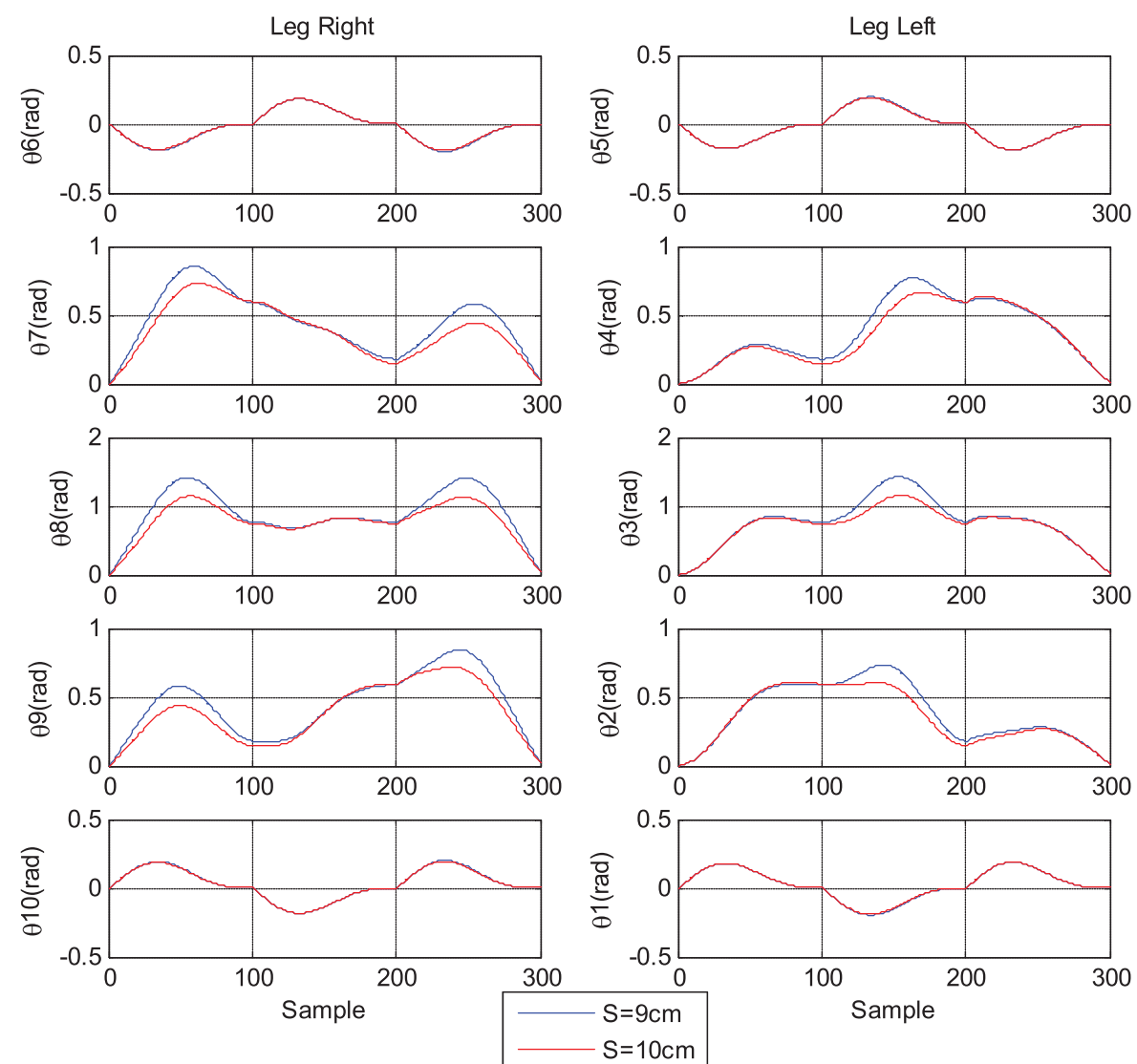

Figure 17 Trajectories of the ten joint angles located at two legs of biped HUBOT-4.

\section{AUTHORS' CONTRIBUTIONS}

Anh H.P.H.: conception, final rewriting, corresponding. Huan T.T.: software, draft writing, data collecting and checking.

\section{ACKNOWLEDGMENTS}

This research is fully funded by National Foundation for Science and Technology Development (NAFOSTED), Viet Nam under grant MDT 107.012018.10 .

\section{REFERENCES}

[1] W.T. Miller III, Real-time neural network control of a biped walking robot, IEEE Control Syst. Mag. 14 (1994), 41-48.

[2] C.L. Shih, Ascending and descending stairs for a biped robot, IEEE Trans. Syst. Man Cybern. 29 (1999), 255-268.

[3] S. Kajita, F. Kanehiro, K. Kaneko, K. Fujiwara, K. Harada, K. Yokoi, H. Hirukawa, Biped walking pattern generation by using preview control of zero-moment point, in Proceedings of the IEEE International Conference on Robotics and Automation, Taipei, Taiwan, 2003, pp. 14-19.

[4] Y.F. Ho, T.H. Li, P.H. Kuo, Y.T. Ye, Parameterized gait pattern generator based on linear inverted pendulum model with natural ZMP references, Knowl. Eng. Rev. 32 (2017), 1-17.

[5] J. Mrozowski, J. Awrejcewicz, Analysis of stability of the human gait, J. Theor. Appl. Mech. 45 (2007), 91-98.
[6] Q. Huang, K. Yokoi, S. Kajita, K. Kaneko, H. Arai, N. Koyachi, K. Tanie, Planning walking patterns for a biped robot, IEEE Trans. Robot. Automat. 17 (2001), 280-289.

[7] V.H. Day, C.M. Chew, A.N. Poo, Optimal trajectory generation for bipedal robot, in Proceedings IEEE-RAS International Conference on Humanoid Robot, Pittsburgh, PA, USA, 2007, pp. 603-608.

[8] G. Dip, V. Prahlad, P.D. Kien, Genetic algorithm-based optimal bipedal walking gait synthesis considering tradeoff between stability margin and speed, Robotica. 27 (2009), 355-365.

[9] M.R. Maximo, E.L. Colombini, C.H. Ribeiro, Stable and fast model-free walk with arms movement for humanoid robots, Int. J. Adv. Robot. Syst. 14 (2017), 1-11.

[10] R. Khusainov, A. Klimchik, E. Magid, Kinematic and dynamic approaches in gait optimization for humanoid robot locomotion, in: K. Madani, D. Peaucelle, O. Gusikhin (Eds.), Informatics in Control, Automation and Robotics, vol. 430, Springer, Cham, Switzerland, 2018, pp. 293-320.

[11] T.T. Huan, H.P.H. Anh, Novel stable walking for humanoid robot using particle swarm optimization algorithm, J. Adv. Intell. Syst. Res. 123 (2015), 322-325.

[12] T.T. Huan, H.P.H. Anh, Stable gait optimization for small-sized humanoid robot using MDE, J. Meas. Control Automat. 21 (2018), 63-74.

[13] T.T. Huan, H.P.H. Anh, Optimal stable gait for nonlinear uncertain humanoid robot using central force optimization algorithm, J. Eng. Comput. 36 (2019), 599-621.

[14] N. Shafii, L.P. Reis, N. Lau, Biped walking using coronal and sagittal movements based on truncated Fourier series, in: 
J. Ruiz-del-Solar, E. Chown, P.G. Plöger (Eds.), RoboCup-2010: Robot Soccer World Cup XIV, vol. 6556, Springer, Berlin, Heidelberg, 2011, pp. 324-335.

[15] E. Yazdi, V. Azizi, A.T. Haghighat, Evolution of biped locomotion using bees algorithm, based on truncated Fourier series, in Proceedings of the World Congress on Engineering and Computer Science 2010 (WCECS 2010), San Francisco, CA, USA, 2010, pp. 378-382.

[16] Y. Farzaneh, A. Akbarzadeh, A. Akbaria, Online bio-inspired trajectory generation of seven-link biped robot based on T-S fuzzy system, Appl. Soft Comput. 14 (2013), 167-180.

[17] D. Gong, J. Yan, G. Zuo, A review of gait optimization based on evolutionary computation, Appl. Comput. Intell. Soft Comput. 2010 (2010), 1-12.

[18] C.F. Juang, Y.T. Yeh, Multi-objective evolution of biped robot gaits using advanced continuous ant-colony optimized recurrent neural networks, IEEE Trans. Cybern. 48 (2018), 1910-1922.

[19] R.V. Rao, Jaya: a simple and new optimization algorithm for solving constrained and unconstrained optimization problems, Int. J. Ind. Eng. Comput. 7 (2016), 19-34.

[20] A. Kumar, V.R. Kumar, S. Datta, S.S. Mahapatra, Application of JAYA algorithm for the optimization of machining performance characteristics during the turning of CFRP (Epoxy) composites: comparison with TLBO, GA, and ICA, Eng. Comput. 33 (2017), 457-475.
[21] R.V. Rao, et al., A new multi-objective Jaya algorithm for optimization of modern machining processes, Adv. Prod. Eng. Manag. 11 (2016): 271-286.

[22] R.K. Suraj, G. Subhojit, Jaya based ANFIS for monitoring of two class motor imagery task, IEEE Access. 4 (2016), 9273-9282.

[23] M. Bhoye, et al., An emission constraint economic load dispatch problem solution with microgrid using JAYA algorithm, in 2016 International Conference on Energy Efficient Technologies for Sustainability (ICEETS), IEEE, Nagercoil, India, 2016, pp. 497-502.

[24] R.V. Rao, A.A. Saroj, Self-adaptive multi-population based Jaya algorithm for engineering optimization, Swarm Evol. Comput. 37 (2017), 1-26.

[25] D.C. Du, et al., Efficiency of Jaya algorithm for solving optimization-based structural damage identification problem based on a hybrid fitness function, Eng. Optim. 50 (2018), $1233-1251$.

[26] D.D. Cong, et al., Damage assessment in plate-like structures using a two-stage method based on modal strain energy change and Jaya algorithm, Inverse Probl. Sci. Eng. 27 (2019), 166-189.

[27] R.V. Rao, K.C. More, Design optimization and analysis of selected thermal devices using self-adaptive Jaya algorithm, Energy Convers. Manag. 140 (2017), 24-35.

[28] R.V. Rao, A. Saroj, Constrained economic optimization of shelland-tube heat exchangers using elitist Jaya algorithm, Energy. 128 (2017), 785-800. 